\title{
Simulation of Multiangular Remote Sensing Products Using Small Satellite Formations
}

\author{
Sreeja Nag, Charles K. Gatebe, and Thomas Hilker
}

\begin{abstract}
To completely capture the multiangular reflectance of an opaque surface, one must estimate the bidirectional reflectance distribution function (BRDF), which seeks to represent variations in surface reflectance as a function of measurement and illumination angles at any time instant. The gap in angular sampling abilities of existing single satellites in Earth observation missions can be complemented by small satellites in formation flight. The formation would have intercalibrated spectrometer payloads making reflectance measurements, at many zenith and azimuthal angles simultaneously. We use a systems engineering tool coupled with a science evaluation tool to demonstrate the performance impact and mission feasibility. Formation designs are generated and compared to each other and multisensor single spacecraft, in terms of estimation error of BRDF and its dependent products such as albedo, light use efficiency (LUE), and normalized difference vegetation index (NDVI). Performance is benchmarked with respect to data from previous airborne campaigns (NASA's Cloud Absorption Radiometer), and tower measurements (AMSPEC II), and assuming known BRDF models. Simulations show that a formation of six small satellites produces lesser average error $(21.82 \%)$ than larger single spacecraft $(23.2 \%)$, purely in terms of angular sampling benefits. The average monolithic albedo error of $3.6 \%$ is outperformed by a formation of three satellites $(1.86 \%)$, when arranged optimally and by a formation of seven to eight satellites when arranged in any way. An eight-satellite formation reduces albedo errors to $0.67 \%$ and LUE errors from $89.77 \%$ (monolithic) to $78.69 \%$. The average NDVI for an eight satellite, nominally maintained formation is better than the monolithic 0.038 .
\end{abstract}

Index Terms-BRDF, constellations, CubeSat, multi-view measurements, NDVI.

\section{INTRODUCTION}

I $\mathrm{N}$ order to completely capture the reflectance of an opaque surface, one must measure the bidirectional reflectance distribution function (BRDF) [1]-[3]. BRDF is influenced by intrinsic properties of the reflecting surface, which are not affected by the position of the sensor relative to the surface, the choice of the coordinate system, and the particular parameterization of

Manuscript received January 13, 2016; revised April 28, 2016; accepted May 13, 2016. The work of S. Nag was supported by the Schlumberger Foundation's Faculty for the Future Fellowship and the NASA Earth and Space Science Fellowship. (Corresponding author: Sreeja Nag.)

S. Nag is with the NASA Goddard Space Flight Center and Bay Area Environmental Research Center, Petaluma, CA 94952 USA (e-mail: sreejanag@alum.mit.edu).

C. K. Gatebe is with the NASA Goddard Space Flight Center and Universities Space Research Association, Greenbelt, MD 20771, USA (e-mail: charles.k.gatebe@nasa.gov).

T. Hilker is with the University of Southampton, Highfield Rd Southampton, SO17 IBJ, United Kingdom (e-mail: T.Hilker@ soton.ac.uk).

Color versions of one or more of the figures in this paper are available online at http://ieeexplore.ieee.org.

Digital Object Identifier 10.1109/JSTARS.2016.2570683 the surface and can be measured within the surface itself without any reference to a larger space. BRDF itself, as a ratio of infinitesimals, is a derivative with instantaneous values of reflected radiance and solar illumination [1]. While it can never be measured directly, BRDF can be approximated from measurements that involve tiny but nonzero changes in illumination and reflectance angles, including laboratory setups [2], [4], [5]. BRDF of a ground spot can be estimated from air or space, using multiple images over the same area viewed from different directions at a specific angle of solar illumination.

The uncertainties caused by surface reflectance anisotropy as a result of changing view-illumination geometry makes it extremely difficult to perform dense angular sampling of the same ground spot at the same time. Single spacecraft have traditionally approximated the angular samples by combining measurements taken over time with forward-aft (e.g., MISR [6] or MultiAngle Imaging Spectroradiometer on the Terra spacecraft which obtains spectral radiance images from nine different directions $\left(0^{\circ}, \pm 26.1^{\circ}, \pm 45.6^{\circ}, \pm 60.0^{\circ}\right.$, and $\left.\pm 70.5^{\circ}\right)$ or cross-track swath (e.g., MODIS [7] or moderate-resolution imaging spectroradiometer on Terra which accumulates multiangular data on subsequent orbits) or precommanded autonomous pointing (e.g., CHRIS [8] or compact high-resolution imaging spectrometer on Proba- 1 that provides spectral radiance images at five angles $\left(0^{\circ}, \pm 36^{\circ}\right.$, and $\pm 55^{\circ}$ in the along track direction) sensors. However, a single satellite without autonomous pointing can make measurements only along a restrictive plane with respect to the solar phase. For example, MODIS collects measurements at solar illumination angle differences of up to $20^{\circ}$ from edge to edge of its swath, with additional variation due to latitude and time of the year [9]. Angular measurements made by single satellite are separated in time by minutes alongtrack (e.g., MISR) or days/weeks cross-track (e.g., MODIS). In areas of fast changing surface or cloud conditions especially during spring melt or tropical storms, a few days can make a large difference in reflectance. While a single satellite with autonomous pointing, such as CHRIS on Proba- 1 , provides the angular coverage unrestricted to a single plane within a few minutes, it cannot provide global, repeatable coverage because of its command-and-control pointing that is focused on specific targets only.

Airborne instruments such as the Cloud Absorption Radiometer (CAR), NASA's heritage radiometer for estimating BRDF [2], [3], [54] can sample only a few locations at specific times of the year when a campaign is commissioned. A spaceborne mission is required for global, repeatable BRDF estimations. Distributed space missions (DSMs) have the potential to provide a middle ground between the angular sampling of single 


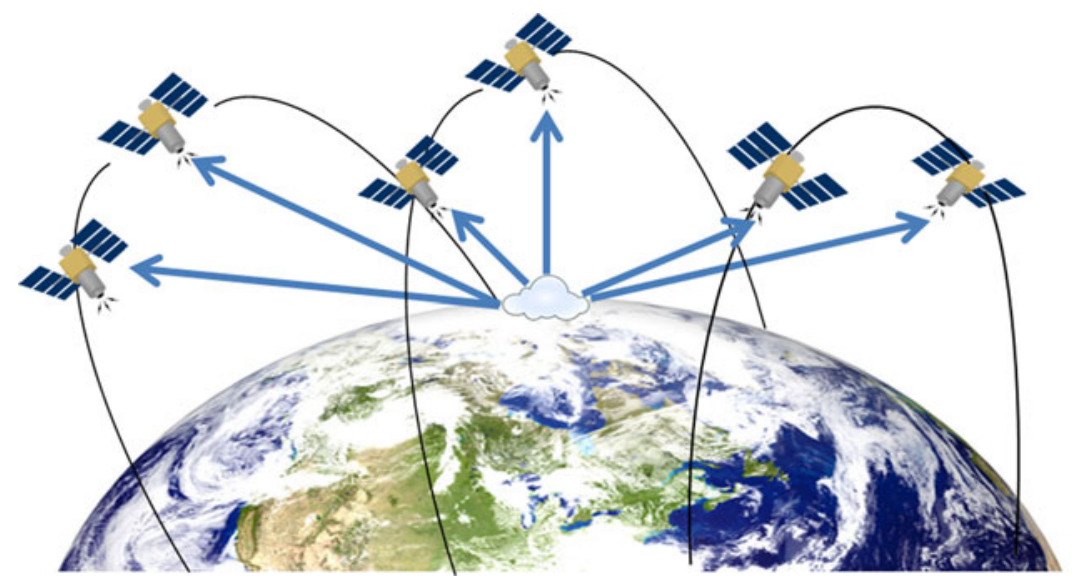

Fig. 1. Formation or cluster of six satellites in arranged within and over three orbital planes at the same altitude and inclination, pointing to and imaging the same ground spot simultaneously

aircraft and global sampling of single spacecraft. A formation of small satellites can make multispectral measurements of a ground spot at multiple angles at the same time as they pass overhead using narrow field of view $\left(\mathrm{FOV}<3^{\circ}\right)$ instruments in controlled formation flight [10]-[12]. Fig. 1 shows a graphic for a six-satellite case, where the relative positions of the satellites do not need to be tightly controlled, but their relative attitudes do. Near-simultaneous angular measurements of the same ground spot eliminate inaccuracy due to surface inhomogeneities or the changing solar angles. There will be a significant difference in the pixel spatial resolution for images acquired when tilting sideways compared to when pointing nadir, however the challenge to integrate such multiangular images exists even when the images are acquired using the same, single platform; therefore, the same integration algorithms can be leveraged.

DSMs have only recently been proposed for or implemented in large scales in government[13] (e.g., NASA's Earth Science Technology Office 2030 Science Vision envisions "distributed observations" and formation flight), academia (e.g., Europe's QB50 mission [14]) and industry (e.g., Planet Labs, [15] and Skybox, [16]) to address the need for repeated, global measurements for Earth observations, monitoring and quick response. NASA's decadal surveys or their mid-term assessments have called for the consideration of DSMs in areas of Earth science, Astrophysics, Heliophysics, and Planetary science. The advent of the CubeSat standard, miniaturization of propulsion, power systems or electronics, frequent and cheaper launch opportunities by emerging companies such as SpaceX and RocketDyne, hosted payload opportunities on traditional rockets using the Evolved Expendable Launch Vehicle Secondary Payload Adapter, deployment mechanisms for CubeSat payloads using the Poly-Picosatellite Orbital Deployer or NanoRacks and the increasing availability of ground stations [17] has made the deployment and operation of large numbers of small satellites more feasible than it ever used to be. In fact, larger numbers are financially feasible because smaller satellites are possible [18].

DSMs have been critical to the success of science goals, otherwise not possible with single spacecraft. For example, GRACE for gravity estimation [ 19], STEREO for three-dimensional
(3-D) imaging of the Sun [20] and the upcoming CYGNSS for wind speed measurements [13]. DSMs can increase data redundancy and thereby provide a clear way of quantifying errors in parameter estimation and identifying major biases or flaws from single sensors. For example, the combined cloud information from CALIPSO and CloudSat has exposed significant biases in interpretation of International Satellite Cloud Climatology Project global cloudiness, led to a new and more accurate way of retrieving aerosol optical depth and yielded powerful new information about polar stratospheric clouds [21]. The benefits of multilook instruments to provide denser angular sampling are well recognized in BRDF products compared to single look [1], [6], [7], [22], however, DSMs have never been developed for them. This paper quantifies the benefits of using formation flight for BRDF estimation when compared to multisensor approaches on a single spacecraft.

Designing DSMs is challenging because the process includes a much larger number of variables than single spacecraft design, and it is imperative to understand the tradeoffs and interdependences among the variables early in the design stage. Model-based systems engineering (MBSE) is a useful tool for preliminary mission concept designs that has been used for many large missions in the past and has recently demonstrated success in small satellite design [23] for understanding the tradespace between conflicting design variables. Previous research on distributed satellite missions [24], [25] has treated satellite systems as modular information processing centers, used N2 diagrams to represent the iterative flow of information between subsystems and optimized performance for extremely simple metrics such as revisit time. Complex metrics specific to missions can be calculated using observing system experiments (OSE) or simulated using observing system simulation experiments (OSSE). $\mathrm{OS}(\mathrm{S})$ Es have been traditionally used to quantify the impact of observations from future observation systems such as satellite instruments or ground-based networks on data products such as weather forecasts, by mimicking the process of data assimilation to validate science return for proposed instruments [26]. OSSEs are important for effective DSM design in Earth science, because the quantitative gaps of flagship missions can only be 
established in the language of specific performance metrics, inherent to the flagship mission concepts. This paper integrates MSBE with OSSE and uses the coupled tool to identify DSM designs that perform better than multisensor, single spacecraft for BRDF estimation, purely from an angular sampling standpoint. Design architectures can be generated from unique combinations of orbits, satellite number, etc., technically assessed using MBSE and the value of those architectures in terms of data assimilation and science products can be assessed using an OSSE. Typical OSSEs are very resource-intensive, therefore, a simple OSSE for differentiating between different DSMs in terms of BRDF retrieval errors has been proposed in [12] and will be used here to demonstrate the utility of DSMs in BRDF estimation. The coupled technique simultaneously addresses the current gap in MBSE, which lacks science evaluation and the current gap in OSSEs which evaluates only point designs.

\section{ApplicAtions of Global BRDF Estimation}

The accuracy of BRDF estimation has an impact on the accuracy of estimating many Earth science products (e.g., albedo, and vegetation indices), especially for non-Lambertian surfaces. The impact of BRDF estimation in terms of only angular dependence of reflectance on the albedo, photosynthetic efficiency, and vegetation indices will be discussed in this paper. The sections below describe the said products and provide a review of literature that supports the need for improved multiangular measurements for their improved accuracy.

Multiangular imaging by Earth observation satellites is considered important for estimating surface albedo, which is a key controlling geophysical parameter needed in the surface energy budget studies, numerical weather forecast, and general circulation models. Albedo is related to land surface reflectance by directional integration of reflectance measured over the full viewing hemisphere at a single or over all solar zenith angles and is therefore dependent on the BRDF, which describes how the reflectance depends on view and solar angles [1]. As early as 1998, the NASA ARMCAS airborne campaign [22] in Alaska measured reflectance at thousands of zenith and azimuth angles using a radiometer that was flown around in circles on an airplane and estimated albedo using these hemispherical measurements. The comparison with nadir reflectance albedo shows $45-50 \%$ errors depending on the wavelength or geolocation sampled. A more recent study [27] shows 15-20\% difference between vegetation albedo estimated using many angles - tower or aircraft and MODIS albedo products.

The Earth's albedo has been an important component of climate studies and the Earth radiation budget since the 1960s [28]. Vonder Haar and Suomi [28] established that Earth's albedo was closer to 0.3 and not 0.4 as was previously thought, but further progress has not been made on albedo uncertainty. A change of 0.01 in albedo corresponds to a $3.4 \mathrm{~W} / \mathrm{m}^{2}$ change in reflected or absorbed sunlight (assuming outgoing radiation to be 341.3 $\mathrm{W} / \mathrm{m}^{2}$ [29]) which is more than half the Earth Radiation Imbalance [30], as will be described later. Climate modeling requires albedo with an absolute accuracy of 0.05 , according to [31], and of 0.02 according to [32], therefore it is important to not only reduce the average albedo estimation error from measurements but also the maximum error below the stated values.

Accurate BRDF is required in quantifying the extent to which forests and vegetation act as a sink for atmospheric carbon dioxide, which in turn is imperative to estimate carbon feedbacks of vegetation in response to global climate change [33]. Deforestation and forest degradation accounts for $12 \%$ of anthropogenic carbon emissions, which have nearly doubled in the past 30 years [34]. Vegetation analysis is adversely affected by undersampling on the principal plane and hotspots [35]. Current gross primary productivity (GPP) estimates show uncertainties up to $40 \%$ in the terrestrial carbon uptake [36] — known as the missing carbon problem over land. GPP is the product of photosynthetic efficiency $(\varepsilon)$ —also called light use efficiency (LUE) — and absorbed photosynthetically active radiation (APAR). In recent studies, it has been shown that measurements of vegetation reflectance at multiple angles can be used to estimate changes in protective leaf pigments as a function of shadow fraction [37]. These protective leaf pigments (xanthophylls) regulate LUE in leaves: Under conditions where factors other than light are limiting the photosynthetic processes, excess radiation energy is dumped as heat, a process which strongly depends on incident solar radiation. This down regulation can be measured by means of the photosynthetic reflectance index (PRI), a normalized difference index that is sensitive to the xanthophyll absorption at $531 \mathrm{~nm}$. Photosynthetic efficiency is proportional to the differential of PRI with respect to the shadow fraction [38]. This differential can be estimated from the BRDF of PRI. There is no correlation between PRI, not corrected for BRDF, and efficiency and the no mapping inferences can be made between the two.

Recent studies have also suggested an overestimation of the greening of Amazon forests during the dry season due to seasonal artifacts in MODIS' sun-sensor geometry and therefore inaccurate BRDF estimation [39]. Using denser, space-based angular sampling (using CHRIS instrument mounted on Proba1, dynamic pointing satellite [6]) reduces GPP uncertainties to $10 \%$ [37], showing a $75 \%$ improvement in carbon cycle calculations. However, Proba-1's measurements were on demand and repeatable, global measurements were not possible.

Normalized difference vegetation index (NDVI) is the ratio of the difference of the reflectance at near infrared (NIR) and red bands to the sum of the reflectance at the same bands [40]. MODIS provides NDVI at different spatial resolutions, at different frequencies ( $250 \mathrm{~m}$ every 16 days to $25 \mathrm{~km}$ NDVI monthly) and serves as a continuity index to NOAA-AVHRR-derived NDVI (approximately 9 day cycle) [9]. NDVI can be used as a vegetation monitoring tool. The temporal profile of NDVI has been shown to depict seasonal and phenologic activity, length of the growing season, peak greenness, onset of greenness, and leaf turnover or "dry-down" period. Ratioing of the NIR and red spectral bands in a normalized manner to compute vegetation indices does not remove surface anisotropy effects in estimation errors, unless the angular variation of the BRDF function at both bands is exactly the same [41]. NDVI errors are dependent not only on the spectral BRDFs themselves, but also their relation to each other and NDVI values get even more distorted due to longer atmospheric path lengths associated with off-nadir 


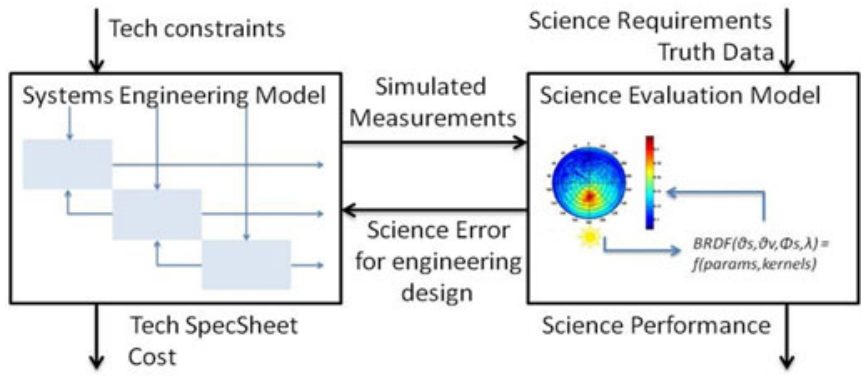

Fig. 2. Coupled formation design (driven by MBSE) and evaluation (driven by science-inspired OSSEs) tool for designing a formation for bi-directional reflectance estimation from space.

viewing. BRDF knowledge is needed for successful utilization of vegetation indices and the derivation of land cover-specific biophysical parameters [42] and BRDF corrections of AVHRR reflectance to a standard nadir view angle have been shown to improve the accuracy of the composited NDVI [41], [42]. Aerosol retrievals are also affected due to inadequate scattering angles in polarization data [6], [43]-[45].

\section{COUPLED MBSE AND OSSE METHODOLOGY}

An architecture is defined as a unique combination of design variables such as number of satellites, their orbit parameters, spectrometer payload's FOV, imaging mode, etc. The methodology which is employed to assess the optimal architectures and validate their BRDF estimation capabilities couples MBSE with OSSEs. A tradespace of architectures can be analyzed by varying the design variables in the MBSE model and assessing its effect on data assimilation and science products using OSSEs, as shown in Fig. 2. The left-hand box generates architectures, sizes them to check their feasibility and costs them, in keeping with the MBSE approach. The model can be simulated over any time horizon, divided into appropriate time steps. The right-hand box evaluates the angular spread over time for every architecture using a simplified OSSE described in [12] and assesses science performance in terms of estimation errors of BRDF and its dependent products.

DSMs can be made cost efficient if the free-flying elements are small satellites. Small satellites have been endorsed by the U.S. National Research Council as "complement to larger satellites, not a replacement for them" [18]. Small satellites are found to be especially cost effective when their payloads have already been developed elsewhere and the arrangement and deployment of multiple instances of these payloads can be optimized based on mission requirements [18], such as the proposed BRDF formations. The CubeSat form factor is a representative example of a standard nano-satellite bus and CubeSats are known to support multispectral imagers to make Earth reflectance measurements [46]. Therefore, the spacecraft bus requirements within the MBSE model are set to values that adhere to optimistic $6 \mathrm{U}$ CubeSat standards: mass less than $20 \mathrm{~kg}$; physical dimensions within a $6 \mathrm{U}$ bus; and average power less than $40 \mathrm{~W}$. We have not yet sized all the spacecraft subsystems in detail to ensure that it fits exactly in a 6U standard because full spacecraft packaging with structures is out of scope of preliminary concept design (pre-Phase A) and falls within Phase B of the NASA Systems Engineering Lifecycle [47]. If the packaging solution finds $6 \mathrm{U}$ to be infeasible, the spacecraft structure can be expanded to the $12 \mathrm{U}$ standard $(\sim 25 \mathrm{~kg})$ without any loss in relevance of the results presented here.

This paper discusses only the results of the coupled model in terms of angle-dependent science performance (output from the right box of Fig. 2) as a function of the most important architecture-differentiating variables in the engineering design (parameters in the left box of Fig. 2). The selection of the variables and internal optimization of the models have been described in parallel literature [48], [12], [49]. The sections below describe the two models in the coupled framework to provide some context in the selection of the architectures whose performance will be compared to single satellite performance, as validated against reference data (Section IV).

\section{A. Systems Engineering Model (MBSE)}

The MBSE model generates hundreds of formation flight architectures by using BRDF measurement requirements and 6U CubeSat bus requirements as inputs (listed in Fig. 3) and constraints and outputs the following three types of metrics shown in Fig. 3: 1) science performance in green (e.g., signalto-noise ratio or SNR), 2) technology supportability in black, and 3 ) resource measures in red (e.g., cost). The orbits/modes and payload module are the architecture generators while the GNC/ADC (guidance, navigation, and control and attitude, determination, and control), onboard processing, communication and propulsion modules are the architecture feasibility evaluators. In this paper, only angular sampling (as quantified further in the OSSE) will be compared among the different, generated architectures that are deemed technically feasible by the GNC and other subsystems mentioned.

The mission measurement requirements are derived from science goals and specifications of current, successful spaceborne instruments (specifically MISR) and airborne instruments (specifically CAR). Measurement zenith angles up to $80^{\circ}$, measurement azimuth up to $360^{\circ}$, and solar zenith angles up to $80^{\circ}$ are ideal for the full angular spread at any ground spot. Spectral requirements, derived from the CAR [2] instrument, are 14 wavebands with spectral resolution varying from 10 to 40 $\mathrm{nm}$ and over the spectral range of 350-2300 nm. These spectral specifications are expected to represent those needed by the passive optical remote sensing community, and can be modified to add new bands or descope existing ones. The spaceborne payload is expected to be a narrow FOV spectrometer, preliminarily outlined in [48]. A medium spatial resolution of less than 500 $\mathrm{m}$ is used as an initial requirement because $500 \mathrm{~m}$ of resolution at the longest required wavelength $(2300 \mathrm{~nm})$ corresponds to a resolution of $188 \mathrm{~m}$ at MISR's highest band ( $865 \mathrm{~nm})$, as per the Rayleigh criterion. Since MISR's resolution at $865 \mathrm{~nm}$ is $275 \mathrm{~m}$, a finer resolution requirement allows for combining data products from the proposed mission with MISR's data products. As with spectral, the spatial requirements can be changed if science products were to change or the other subsystems were to provide such feedback. For example, the payload analysis confirmed a preference to spatial resolutions coarser than $500 \mathrm{~m}$ 


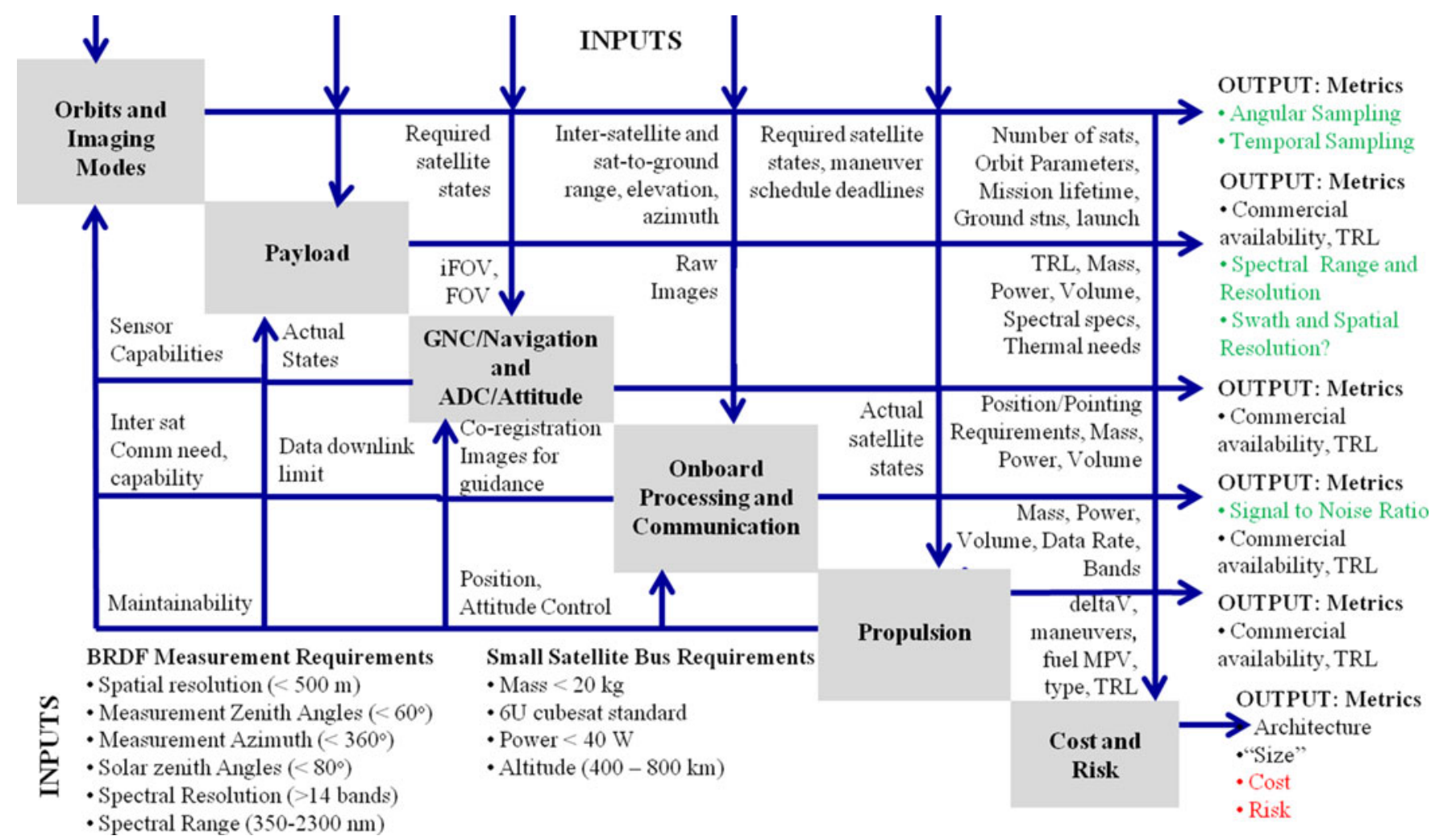

Fig. 3. Systems Engineering Model as an N2 diagram such that the boxes represent the critical modules that drive the technology feasibility study and design of formation architectures to support the science. The vertical arrows represent inputs and the horizontal arrows represent outputs. Green outputs indicate science performance or serve as inputs into the science evaluation box in Fig. 2; red outputs indicate cost; black outputs indicate technical performance. The text in the N2 diagram represents the variable flow between the two modules connected by a bent arrow, where in the text is placed at the corner of the arrow's right angle. MPV = Mass, Volume, Power.

in order to improve swath for more coverage and allow more integration time per image, thus more SNR. The altitude range is constrained to $400-800 \mathrm{~km}$ (LEO) because that range corresponds to the most common shared rides available with primary payloads. If all the satellites can be launched as the primary payload itself, then the orbit constraints may be removed. The given LEO range has also been found to be a sweet spot for payload operations and maintenance against atmospheric drag.

The flow of information between the modeled subsystems is shown in Fig. 3 as an N2 diagram (defined in [24], [25]) so that only feasible architectures are outputs from the MBSE model, to be evaluated by the OSSE. In keeping with the structure of N2 diagrams, the arrows on the upper and lower triangle represent feed-forward and feedback loops, respectively. Information flow is represented as lists of variables between any two modules, where in the text is placed in the knee or corner of the right-angle arrow connecting the two modules. For example, the GNC/ADC module sends "Actual satellite states" to the propulsion module, which sends back "Position, Attitude Control" to GNC/ADC and "Maintainability" to the orbits and modes module. The entire penultimate column of variables starting with "number of sats" are variables that flow from all modules into the cost and risk module.

The functions of each module, in the context of simulating and evaluating DSM architectures, are briefly described below. The orbits and imaging modes module generates formation flight architectures that satisfy the input constraints and measurement requirements. Since there are hundreds of thousands of possibilities, the variable space has been streamlined based on semi-analytical sensitivity studies [48] and we compare only the streamlined architectures in this paper. There can be different strategies for payload pointing or imaging modes for a given set of orbits. While the orbits module simulate how the satellites will be organized in space, the imaging modes simulate where they will point to capture optimal images in coordination. The pointing strategy chosen for this paper dynamically switches the satellite pointing nadir, and has been shown to maximize performance [49]. The payload module is a physics-based model to find the optical requirements based on external (mission or other module) requirements, use them to design dispersive spectrometers that will fit within a $20 \mathrm{~kg}$ spacecraft, and evaluate their spectral, spatial, and radiometric performance. The FOV and spatial resolution provided by the payload drive the attitude determination and control bits required in the GNC and ADC module. The slew rate of payload pointing required for all the geometry solutions and imaging modes drives the ADC module to find required angular momentum and torque capacity of an appropriate ADC system. If inter-sat communication is used (currently suboptimal [48]), the GNC sensors and range will limit the intersatellite distances allowed in the formations, therefore a feedback loop leads to the orbits module. The onboard processing simulates the intake of raw images from the 
payload and position information from the GNC module. It processes and compresses the image, ensures accurate image registration so that it can be combined with processed images from other satellites in the formation obtained at the same ground spot at different angles. The processed image may be used to further enhance the spacecraft's state knowledge. The communication module is responsible for simulating the transmission of the processed images down to the Earth at the next downlink opportunity as a function of the range and elevation to ground station (as obtained from GNC). Limited by available data rate and ground stations, the processing and communication modules set a limit in terms of the amount of data that can be sent to Earth, which in turn sets a limit (feedback loop) on the data collection rate of the payload. The propulsion module simulates the initialization of the formation after launch, station-keeping and momentum dumping for the ADC system. If maintenance within current capability for small satellites is not possible for an architecture's orbits, as evaluated by the propulsion module, that particular architecture will be discarded. The cost and risk module is used to cost every architecture including its complexity and risks to estimate tradeoffs against the science performance of that architecture.

Many formation architectures were generated by running the MBSE model for different subsystem variables within measurement requirements. Technical feasibility of these architectures were determined by the output values, shown in black text on the right-hand side of Fig. 3, as modeled by the relevant modules: SNR, commercial or off-the-shelf availability of technology required for the payload, GNC/ADC, communication, onboard processing and propulsion subsystems and their corresponding readiness level (TRL). Science performance of these architectures was determined by the output values in green text: angular spread, spectral range and resolution, spatial resolution, and swath and temporal resolution. Of these, angular spread served as the input into the Science Evaluation model, because it cannot be evaluated in absolute terms like the other metrics. Only architectures with the values permitted by the technology constraints and measurement requirements were considered. For example, technologies required by the GNC/ADC module (e.g., star tracker accuracies) were checked against industrial catalogues for small satellites and whether the downlink channels for the required data rates could be obtained. If not, either the relevant architectures were discarded, or the measurement requirements relaxed (e.g., payload collection rate reduced). This paper will not cite or compare costs of different options, because a single-unit cost depends on the detailed satellite design and the design, cost to copy multiple units and large-scale operations depends on the mission contractor. Instead, we will show performance impact, in terms of BRDF and dependent products, as a function of increasing number of satellites and, by extension, resources.

\section{B. Science Evaluation Model (OSSE)}

Airborne or tower data of multiangular reflectance (reflectance at all view and relative azimuth angles at $1^{\circ}$ resolution) serve as "Reference BRDF" for the science evaluation model. The BRDF angular pattern is dependent on the wavelength and surface type [2]. Therefore, reference BRDF to be used at any instant in the simulation depends on the geographic area that the formation is expected to fly over and the data products of interest. The simplified OSSE model evaluates each architecture in the following way: At any simulated instant of time, a subset of BRDF values, that correspond to the view zenith and azimuth angles of the formation's satellites at that instant, is selected from the "Reference BRDF" and used as data to invert a BRDF model and estimate the model parameters. These parameters are then used to run the forward model and compute simulated reflectance at all angles at that time instant. The difference between this estimated reflectance and the reference CAR reflectance is called the "BRDF error" and is represented as a root mean square value (RMS) over all 90 view zenith multiplied by 360 relative azimuth angles. BRDF can be used to calculate geophysical variables such as albedo and GPP. The difference between these variables calculated from the "reference BRDF" reflectance values versus the forward model estimated values is called the application error, for e.g., albedo or NDVI error. BRDF errors and application errors at every instant of time in the simulation are the outputs from the science performance evaluation model and determine the goodness of the input formation design and corresponding angular spread. By calculating the error over time for a full tradespace of formation architectures or designs helps us judge them based on an intricately coupled science metric.

The error obtained using the above algorithm is a sum of the model error, linear inversion errors, and angular sampling error. Since the first two are found to be negligible [12], the error term represents the goodness of angular sampling of the "Reference BRDF." The simulated or reference data in our models do not include atmospheric corrections because they are not expected to cause significant differences in the BRDF errors between the different architectures, when compared to errors due to angular sampling. The "acquired" simulated images are not screened based on expected cloud cover (40-50\%) because clouds are not architecture-differentiating either and adding clouds would impact global coverage more than local, angular coverage of a specific image. Global coverage can be improved by adding more formations, not within the scope of this paper. Combining off-nadir images with nadir ones to generate multiangular products implies combining different pixel sizes. The BRDF characteristics of large pixels are not necessarily the same as those of small pixels, however the current MODIS and MISR algorithms already integrate nadir and off-nadir measurements, therefore the pixel size distortion is not seen as a problem newly or additionally introduced due to small satellite formations. The BRDF parametric models chosen for the OSSE are the RossThick Li-Sparse (RTLS) model [50] over land and the CoxMunk model [51] over water. They were preferred over other models such as the RPV, modified RPV, and Walthall models based on sensitivity studies [12].

1) Airborne Data From CAR: Local BRDF data from airborne campaigns of the CAR on platforms such as NASA P-3B is used as "truth" or "reference" [2] in BRDF calculations in all applications except LUE estimation. The CAR has an instantaneous FOV (iFOV) of $1^{\circ}$ and is designed to have a zenith to nadir scan range of $190^{\circ}$. By flying it around a ground spot in circles 


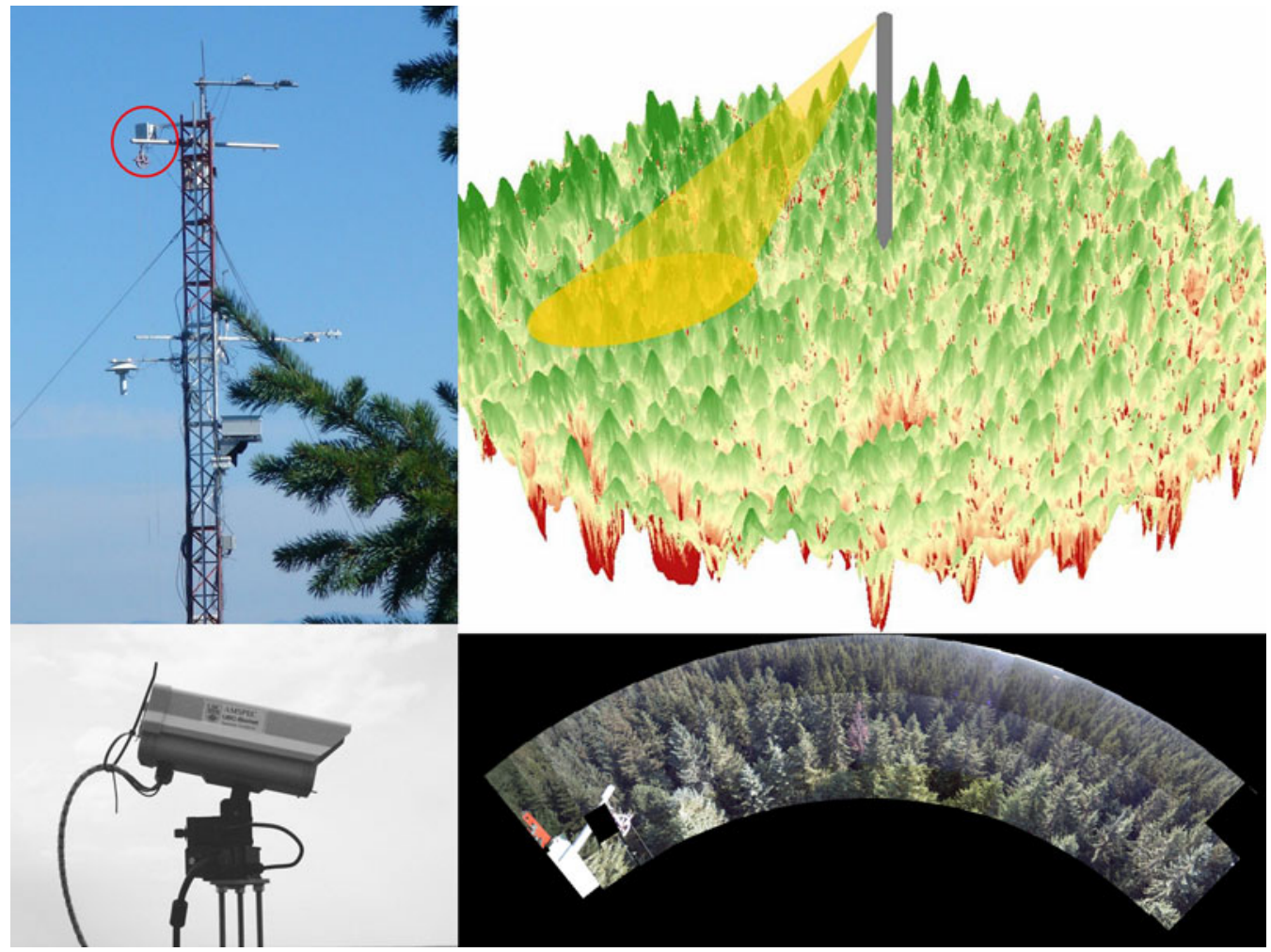

Fig. 4. AMSPEC instrument (bottom left), mounted on a tower for field tests (top left). The instrument can spin and tilt to adjust its azimuth and elevation respectively (top right), producing multi-angular images of the area around the tower (bottom right).

and at different heights, radiance measurements are obtained at every degree of reflectance zenith (up to $90^{\circ}$ in elevation angles) and $360^{\circ}$ azimuth angles for state-of-art BRDF estimation. By repeating measurements at different times of the day, reflectance at different solar zenith angles may also be available but it is not as exhaustive as the measurement angles.

Seven major surface types have been assumed for the study: water, snow/ice, savannah, croplands, desert, forests, and cities [12]. Their global distribution was extracted from the Global Land Cover Facility, which draws from MODIS data. The global grid points are $5^{\circ}$ apart at the Equator and distance-adjusted for higher latitudes. The use of surface types in this study is also based on the broad directional features of BRDF described in [2], obtained from 1991 to the present, where the measurements have been acquired from six different aircraft and over four continents. For each surface type, CAR data from airborne campaigns were retrieved from the NASA GSFC database and geometrically corrected reflectance stored as a function of measurement zenith and azimuth with respect to the Sun. Both the shape and the intensity values are very different for each surface type, indicating the importance of local but angular data collection as well as global and temporal assessment of this data.

2) Tower Data From AMSPEC II-The Automated Multiangular Spectroradiometer: Canopy reflectance measurements from an automated, multiangular, spectroradiometer platform called AMSPEC [52] is available for some forested regions. These measurements are used as reference BRDF at the $531 \mathrm{~nm}$ band, which the CAR does not possess, and which has been used for calculations of photosynthetic LUE and therefore primary productivity of plants. AMSPEC is a tower-based instrument which can adjust its look angle between $40^{\circ}$ and $70^{\circ}$ and scan up to $330^{\circ}$ of azimuth (after which the tower gets in the way). The mounting and measurement collection of the instrument has been shown in Fig. 4. As an automated instrument, it can collect data all day hence, has more solar illumination angle coverage than the CAR. Note that both AMSPEC II and CAR data, even if available at $1^{\circ}$ angular resolutions are only approximations of BRDF as obtained by state-of-art instruments in field and air, respectively. BRDF is a theoretical ratio of infinitesimals of illumination and radiance [1], therefore can only be estimated from such angular data.

AMSPEC data have been validated to be very closely correlated to the CAR data, and can be used to fill up solar zenith gaps in vegetated or forested regions, if required [48]. This study uses data from a 61-year old, second-growth coniferous forest (hereafter referred to as the DF-49 site) located on Vancouver Island, British Columbia, Canada, at 300-m above sea level (49 $52^{\prime} 7^{\prime \prime}$ $\left.\mathrm{N}, 125^{\circ} 20^{\prime} 6^{\prime \prime} \mathrm{W}\right)$. The stand consists of $80 \%$ Douglas fir, $17 \%$ western red cedar, and $3 \%$ western hemlock and is among the most productive forest types in Canada. The stand density is 1100 stems ha $^{-1}$, with tree height ranging between 30 and $35 \mathrm{~m}$. The site is located within the dry maritime Coastal Western Hemlock biogeoclimatic subzone (mean annual temperature ), which is characterized by cool summers and mild winters with occasional drought during late summer. The leaf area index is $7.3 \mathrm{~m}^{2} \mathrm{~m}^{-2}$ [53]

Since the AMSPEC instrument is mounted on a tower and scans the surrounding canopy by changing its look direction, its angular spread is not as extensive or regular as the CAR instrument. Fig. 5 shows measurement angular spread collected 


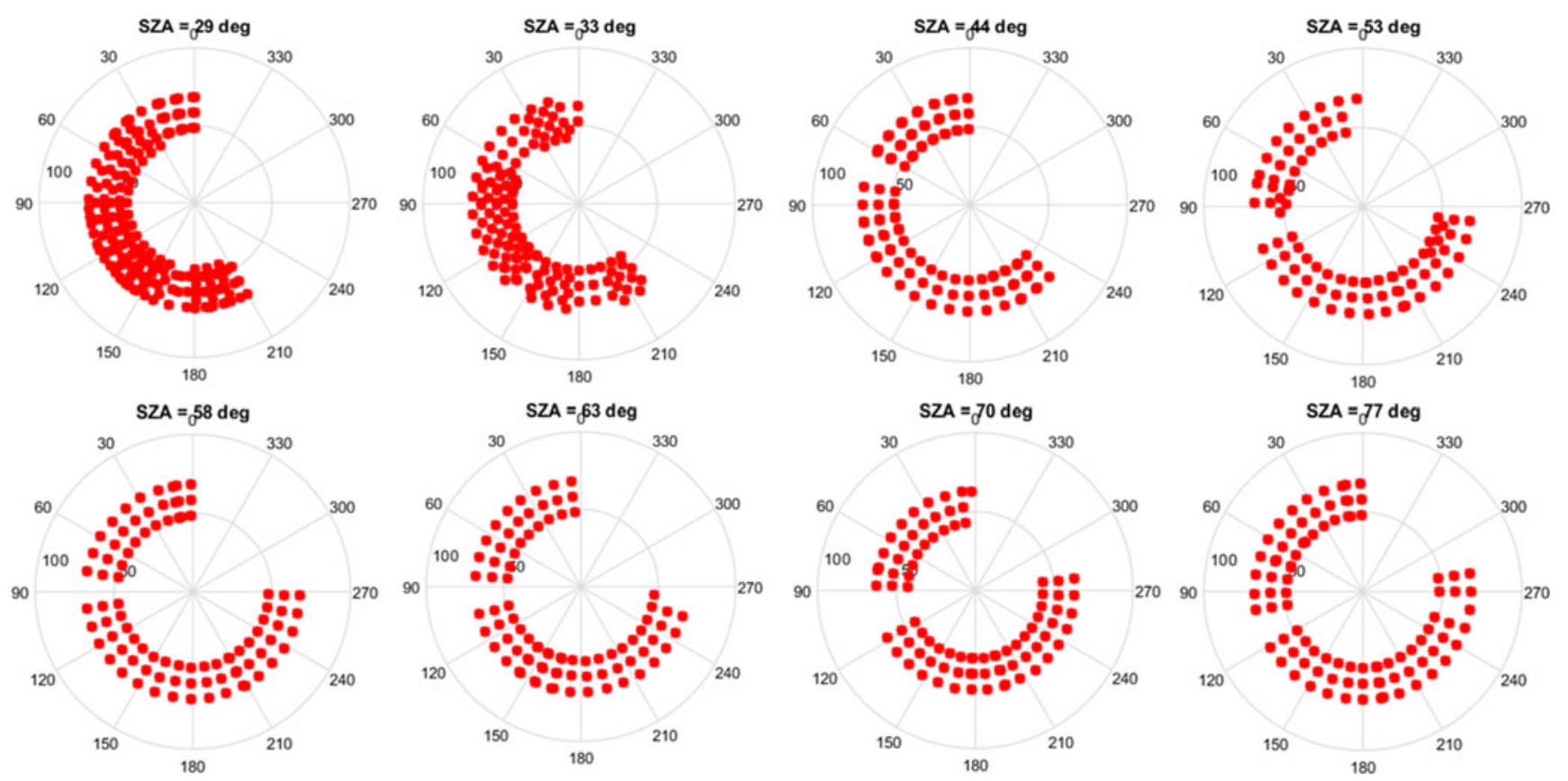

Fig. 5. View zenith (VZA) and relative azimuth (RAA) of the AMSPEC measurements from the DF- 49 site, binned by measurements taken every $7^{\circ}$ of solar zenith (SZA). Radius indicates the VZA and polar azimuth the RAA of the measurement with respect to the Sun.
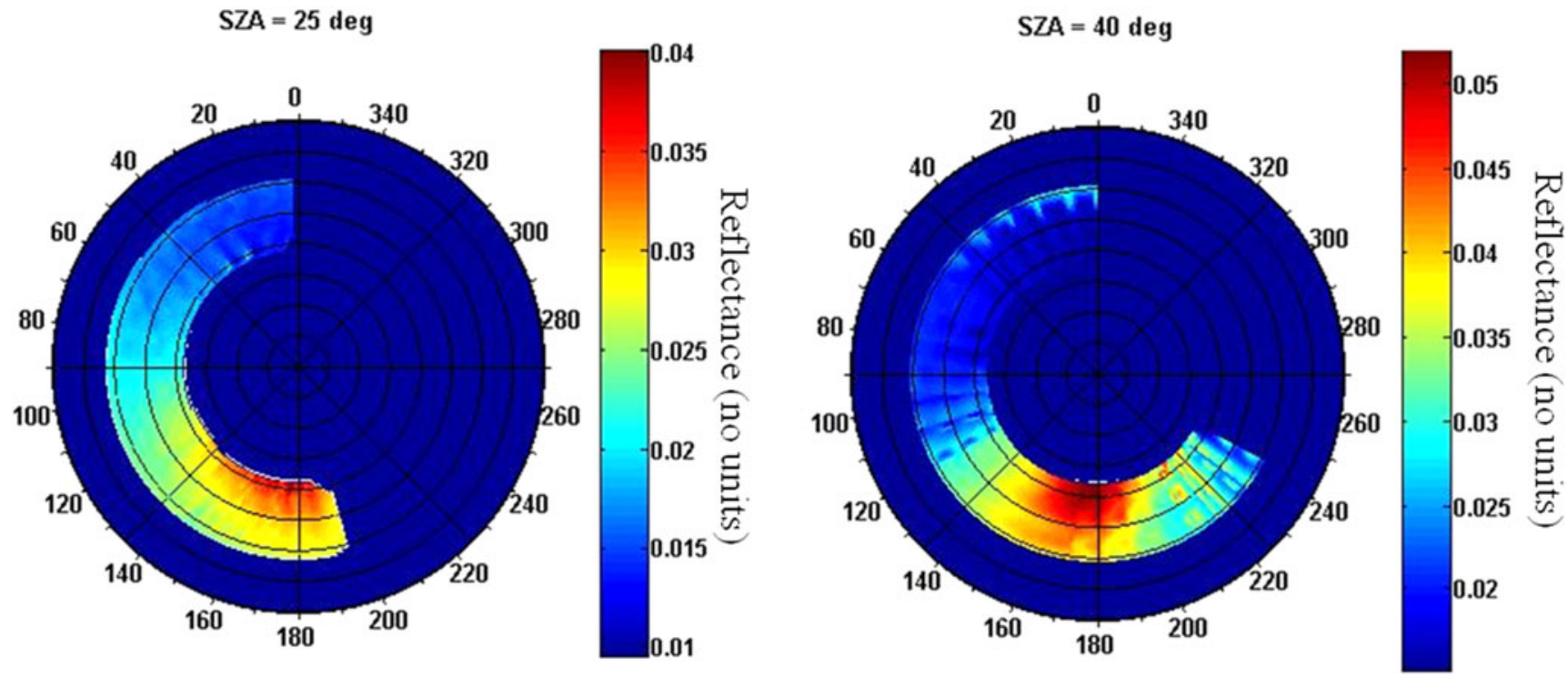

Fig. 6. AMSPEC data at $531 \mathrm{~nm}$ from the DF-49 site as a function of VZA and RAA, for measurements collected at $\mathrm{SZA}=25^{\circ}$ (left) and $40^{\circ}$ (right).

by AMSPEC over a one day span, binned every $7^{\circ}$ of solar zenith angle. The gap in the top, right quadrant is due to the views being obstructed by the tower on which the instrument is mounted. The view zenith range is from $\sim 40^{\circ}$ to $60^{\circ}$ because the instrument cannot look straight down from the tower as CAR can from an airplane. AMSPEC reflectances are binned over short periods of time so that the BRDF values not only capture the directional effects seen in all VNIR wavelengths but also the physiological effect during the xanthophyll cycle (a biophysical mechanism that controls photosynthetic downregulation in leaves), which is more active in sunlit leaves than shaded ones. The reflectance values at $1^{\circ}$ intervals of VZA and RAA for are shown in Fig. 6, at a morning hour in August 2006 during clear sky conditions. The edge of the hotspot and the full hotspot itself is clearly seen in the left and right panels, respectively. All AMSPEC measurements from DF-49 are a conglomerate of measurements over all SZAs, some of which are seen in Fig. 6, because the instrument operates continuously all day. It is clear that this data alone cannot be 

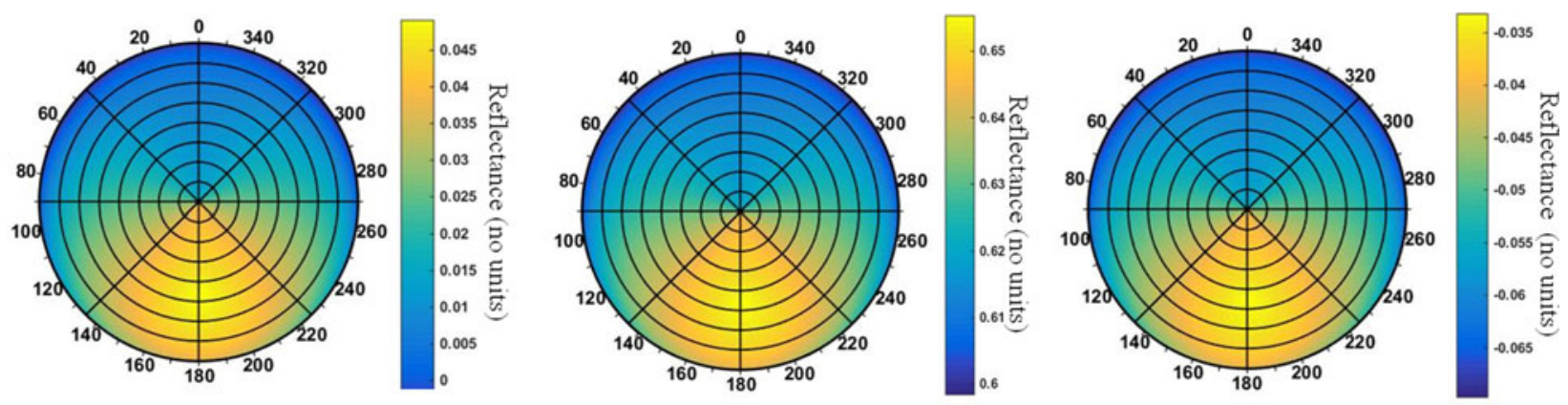

Fig. 7. BRDF of forest data, synthesized by RTLS inversion on data collected over the DF-49 forest site by the AMSPEC instrument in 2006-2009, at the [left] $531 \mathrm{~nm}$ and [center] $570 \mathrm{~nm}$ band. [Right] The corresponding, calculated PRI (always negative). Note the difference in color scales over the three panels.

used as truth because its angular spread is not enough to pick a given random angle that the architectural outputs of the SysEng model produces.

The approach adopted was thus to invert an RTLS model using all the AMSPEC measurements at the $531 \mathrm{~nm}$ band (all VZA, RAA, SZA) and calculate the three RTLS coefficients. These coefficients were then used to calculate reflectance at all VZA and RAA angles, and this reflectance used as the truth or reference for the xanthophyll sensitive band. The same can be repeated for a xanthophyll insensitive band (red band chosen because of its overlap with one of CAR's bands). The BRDF simulated by inverting for and then forward modeling the RTLS parameters is shown in Fig. 7 for both bands. The synthetic data shows $>0.92$ coefficient of correlation with CAR's red band data, validating the applicability of the approach for using sparse, angular data sets as OSSE truth. The PRI calculated from both synthesized data sets is also shown.

\section{Performance Tradeoffs for BRDF-DEPENDENT PRODUCTS}

Angular measurements were simulated for a full day ( $>15$ orbits) using the Systems Engineering Model and errors for BRDF and its dependent products (albedo, LUE, NDVI) computed at every instant using the Science Evaluation Model, depending on the surface type expected to be seen by the formation at that particular instant. One satellite in the formation pointed nadir while the other satellites pointed to the ground spot below the first satellite. The nadir-pointing satellite changed dynamically based on an algorithm documented in [48], [49] because this imaging mode was found to produce the least estimation errors without compromising spatial or global coverage. We assumed that the algorithms for scheduling the nadir-pointing satellite are run and decisions are made in ground stations and communicated to the satellites during daily overpasses. Previous studies have shown that the formation satellites can have only two differences in its orbital elements such that the formation can be maintained with small satellite technology (feedback loop between propulsion and orbits in Fig. 3). For any given number of satellites (say, N) at a specific altitude and inclination, there are ${ }^{8} \mathrm{C}_{\mathrm{N}-1}$ formation architectures available with orbital in-plane and intraplane spacing of approximately $\pm 5^{\circ}$ (chosen because a few degrees of difference negligibly affects performance). By extension, for a given altitude-inclination combination, there are a total of 1254 architectures containing 3 to 8 satellites, all of which were compared. Dependence on altitude/inclination of the orbit was found to be negligible because the planar and in-plane separation of the satellites could be changed in order to achieve similar maximum spreads across orbits. Performance depended largely on the number of satellites and how they were arranged, differently for BRDF and its products.

The BRDF error percentage averaged to $23.2 \%$ over multiple orbits, when angular sampling was performed by a single satellite with nine instruments pointing in different angles such as MISR, as compared to CAR reference BRDF at the surface types expected to be below the satellite. It is important to average over several orbits to a day to ensure that all the surface types are accessed by the formation as it orbits the Earth, for a representative error calculation. Six satellites, arranged optimally in a formation, were found to make angular measurements that estimate BRDF better than the MISR arrangement, providing an error of $21.8 \%$. The RTLS model used for BRDF inversion has residual norms $<0.05 \%$, is linear and not dependent on the initial conditions [12], therefore model errors are negligible compared to angular sampling errors. When MATLAB's twosample $t$-test was applied to the error time series for MISR and the best six-satellite formation, it rejected the null hypothesis that the data are independent random samples from normal distributions with equal means and equal but unknown variances at the default $p<0.05$ significance level. Moreover, the sixsatellite formation performed better than MISR's arrangement when the satellites were initialized at different points in the orbital plane, as long as the formation geometry remained intact. To improve the error further at more cost, one can add additional satellites. Eight satellites, arranged in any orientation, as long as the intraplane and in-plane spacing is approximately $\pm 5^{\circ}$ apart and can be maintained as such, were found to estimate BRDF better than MISR's orientation. The best case scenario with eight satellites provided a $21 \%$ error compared to CAR reference data. The following sections describe the performance of satellite formations in terms of a few BRDF-dependent products and the performance comparison with MISR, where estimation errors were calculated using the method discussed in Section III-B. Error due to MISR's expected measurements was calculated using this method instead of using MISR Level 2 Land Surface Data (MIL2ASLS) so that there was no any dependence on BRF noise, inversion errors on aerosol properties 

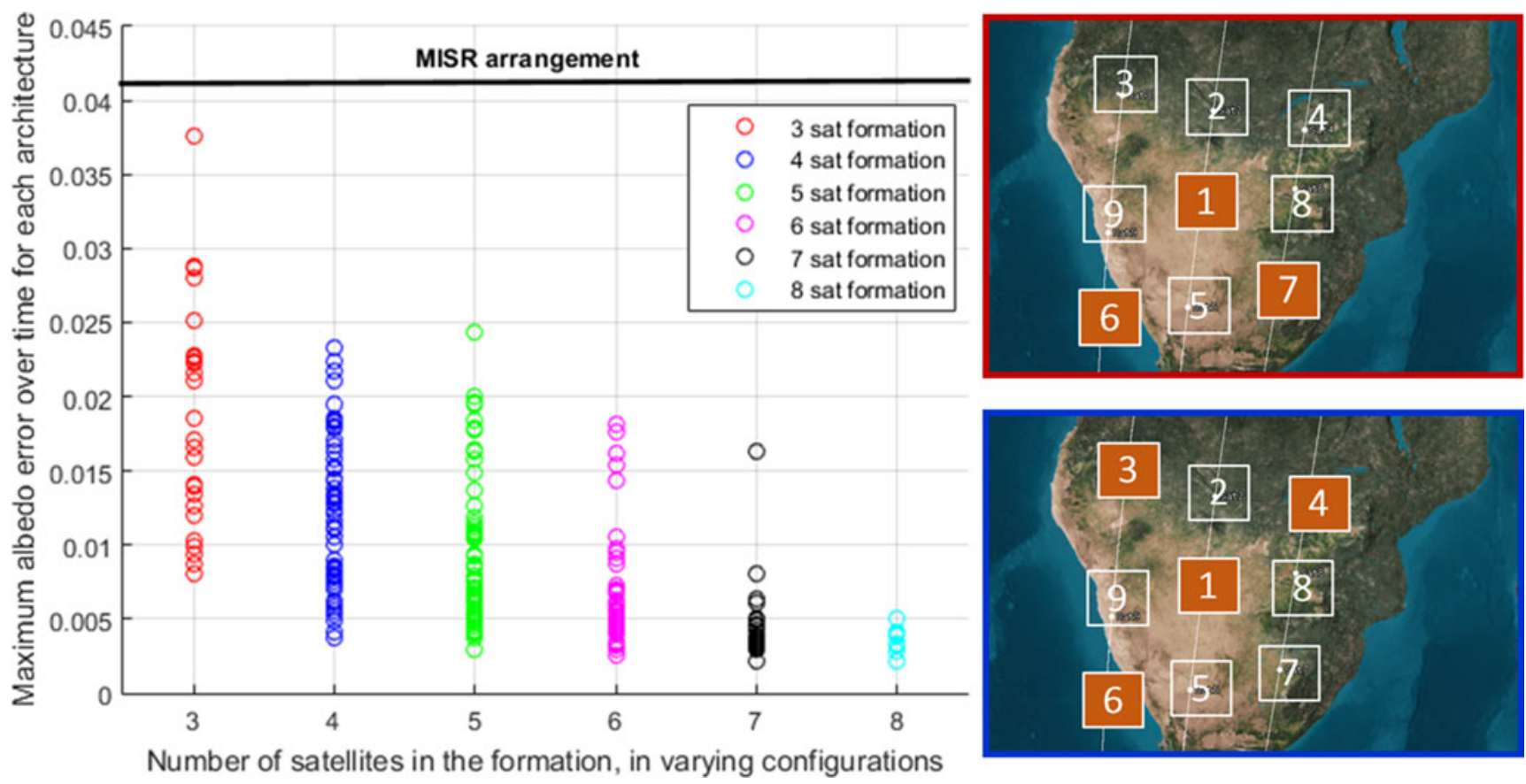

Fig. 8. Maximum albedo error, over simulation time, decreases for increasing satellite number at a $650 \mathrm{~km}, 51.6^{\circ}$ orbit. Each circle represents a different planar and in-plane arrangement between the satellites. The horizontal black line represents MISR's error of 0.042 or $11.9 \%$. When the maximum error is compared as a percentage, it takes five satellites arranged in any slotted formation to outdo MISR's arrangement [49]. Insets correspond to the satellite arrangement with the lowest error of 0.002 and 0.0015 using three (red outline) and four (blue outline) satellites, respectively. The orbital planes are marked by thin, continuous lines.

or any other background noise, and to ensure error comparison with DSMs in terms of only angular sampling.

MISR was chosen as the single spacecraft instrument to compare the small satellite formations against because it is on a repeat track orbit and provides the highest number of near-simultaneous multiangular images (nine images from its nine cameras within ten minutes). All the multiangular measurements simulated for any small satellite formation in this study are simultaneous. Integrating multiday measurements will increase the angular spread of measurements made by a multisensor single satellite or a single satellite formation, however, only those multiangular instruments that provide near-simultaneous angular measurements were found fit for a fair comparison. Other along-track multiangular instruments, such as Along Track Scanning Radiometer-ATSR [29] and the Advanced Spaceborne Thermal Emission and Reflection Radiometer-ASTER [30], provide only two look angles near simultaneously, therefore MISR is a best representation of along-track state-of-the-art. Cross track sensors like MODIS or Clouds and Earth's Radiant Energy System-CERES [27], provide one angular image per ground spot every hour and half, and therefore cannot be considered near-simultaneous. CHRIS provides five look angles per ground spot for every pass, therefore near-simultaneously, which is less than MISR. Moreover, it does not image the same ground spots consistently in time because it is commanded to observe only specific targets. The Polarization and Directionality of the Earth's ReflectancesPOLDER [26] instrument provided 12 angular images due to its wide-angle swath, however its coarse ground resolution of 6-7 $\mathrm{km}$ makes it less useful for the vegetation products discussed in this paper compared to MISR's subkilometer resolution.

\section{A. Impact on Albedo Estimation}

Narrow band albedo was calculated from CAR's reference BRDF data at representative wavelengths (chosen from sensitivity studies [12]) for each of the seven major surface types. Reference or forward modeled albedo were calculated from reference or forward modeled BRDF (after estimating parameters from the simulated angular measurements-Section III-B) by integrating across the reflectance at every one of the 360 relative azimuth and 90 view zenith angles. Albedo error was modeled as the difference between the reference and forward modeled albedo. Since the solar zenith angle for all the CAR datasets was within a few degrees (campaign lasts a few hours daily under clear sky conditions), the calculated product, when used as reference BRDF, could used to estimate black-sky albedo. For our simulations, either the directional-hemispherical reflectance (black-sky albedo) or bihemispherical reflectance (white-sky albedo) could have been used to calculate the albedo for any given illumination conditions, with no loss of generality of the presented results. White-sky is the bihemispherical reflectance under conditions of isotropic illumination, so it has the angular dependency removed. On the other hand, black-sky is the directional hemispherical reflectance computed at local solar noon. In practice, the albedo at a particular solar zenith angle can reasonably be approximated by the proportionate sum of the two terms: black-sky albedo and white sky albedo, which defines the proportion of diffuse illumination.

Comparing the albedo error, averaged over multiple orbits, for satellites deployed in five orbits of differing altitudes and inclinations (raised ISS orbit at $600 \mathrm{~km} / 51.6^{\circ}$, Landsat and A-Train orbit at $710 \mathrm{~km} / 98.2^{\circ}$, Iridium orbit at $790 \mathrm{~km} / 86.4^{\circ}$, 


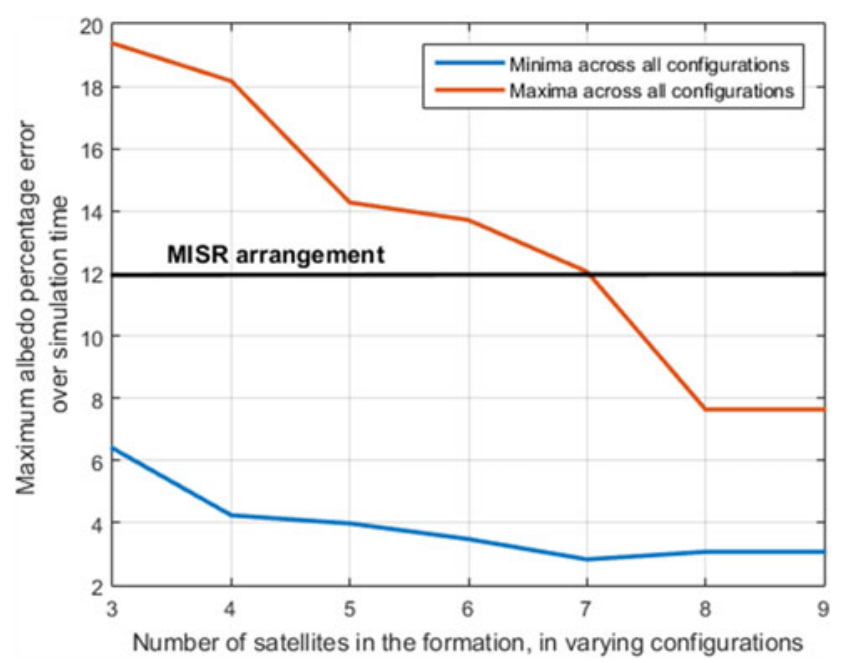

Fig. 9. Average albedo error over time at a $650 \mathrm{~km}, 51.6^{\circ}$ orbit decreases with the number of satellites in the formation (adapted from [49]). Each circle represents a different planar and in-plane arrangement between the satellites. The horizontal black line represents MISR's error of 0.0065 or $3.6 \%$.

sun-synchronous orbit at $600 \mathrm{~km} / 97.79^{\circ}$, sun-synchronous orbit at $425 \mathrm{~km} / 98.2^{\circ}$ ) showed that albedo errors are insensitive to orbital altitude and inclination, as long as all the formation satellites share the same values, allowing the mission designer significant flexibility in choosing any available launch. Note that while the errors presented in Figs. 8 and 9 are average values over multiple orbits, they are derived from the errors calculated at every minute and only for simultaneous measurements. The simulated measurements for MISR's arrangement were assumed to be near-simultaneous to present a fair comparison, even though its nine multiangular images are spread over more than $7 \mathrm{~min}$. Estimates for all formations and single spacecraft are expected to be better, if multiday averaging is used in calculating albedo.

The albedo estimation error for all 1254 formation orientations in an ISS altitude and inclination, calculated at every time step, was averaged over the full simulation time to compare with MISR's configuration. We found architecture options for as low as three satellites that outperform MISR's average error percentage. The range of errors over possible arrangements per satellite number was relatively large. While MISR (time-averaged error of $3.6 \%$ ) was outperformed by some three-sat arrangements (the best error is $1.86 \%$ ), any slip in orbit maintenance or changing the nadir-pointing satellite efficiently could cause the error to shoot above the multisensor single spacecraft design. To mitigate risk and taking into account that the maximum error over all configurations decreases with satellite number, a formation of seven to eight satellites were found to outperform MISR's errors, irrespective of how they are arranged. Formation arrangement was thus as important as number of satellites, but its importance was reduced in the context of operational concerns. Albedo estimation needed lower numbers (three and seven, respectively) compared to BRDF estimation, which needed six and eight satellites, respectively, because albedo is a less rigorous metric by virtue of having only one error term per instant. BRDF error, in comparison, is the RMS value of the error term at every angular direction $(90 \times 360=32400$ values $)$. The highest average albedo estimation error across all shown formation configurations and MISR was 0.005 and 0.003 , respectively, which easily met the absolute albedo error required for climate modeling [32], assuming CAR-measured albedo to be the truth. In reality, there will be many sources of error apart from angular sampling therefore simulated albedo, including all radiative transfer parameters, is likely to be higher than the above numbers.

MISR's BRDF or albedo estimation errors were found to increase significantly, in time, when the nine measurements were made at a relative azimuthal angle perpendicular or close to perpendicular to the solar principal plane. Relative reflectance at the hot spot and sun glint are inadequately measured if the angular space around the principal plane is not sampled. Due to this reason, MISR's maximum albedo estimation error, across all simulation time steps, was more than the maximum error of any of the proposed formation architectures arranged in any way, as seen in Fig. 8. All 1254 formation architectures have been plotted as circles in Fig. 8, color coordinated by the number of satellites in the formation. The in-space formation arrangement of the three and four satellite formation that corresponded to the lowest errors, are highlighted in the inset, as the formation flies over the Southern tip of Africa. As expected, the error and its spread across different configurations decreased with the number of satellites. Three satellites arranged in some configurations and six satellites arranged in any proposed configuration was able to meet the absolute albedo accuracy of 0.02 required by [32] for climate modeling, at any instant of time in their orbits, assuming the albedo measured by the CAR instrument at any given surface type to be the true albedo. The multisensor single-platform architecture (MISR) was able to meet the absolute albedo accuracy of 0.05 required by [31] at all times in its orbit, but it was outperformed by all proposed formation architectures.

Fig. 9 shows a plot similar to Fig. 8, except that the metric on the $Y$-axis is the maximum albedo estimation error for each architecture, expressed as a percentage of reference BRDF (CAR data in Section III-B.1) instead of an absolute value. Errors corresponding to the most (red) and least (blue) erroneous configurations per satellite number are shown, instead of all configurations. It is obvious that the MISR arrangement did comparatively better when percentage was used as a metric, instead of absolute albedo error. Even then, a three-sat formation in some arrangements and a five-sat formation in any of the slotted arrangements outperformed the MISR arrangement. Increasing the number of satellites had noticeably diminishing returns because they were arranged in orbital slots that were well spread in and out of plane. Purely evaluated in terms of angular sampling, MISR's worst performance over time (relative to formations) was far worse than its average performance over time (relative to formations) due to the inflexibility of its geometric arrangement causing the sensors to be badly aligned in at least two periods in every orbit or $\sim 90 \mathrm{~min}$.

\section{B. Impact on LUE Estimation}

Errors in BRDF estimation map into errors in global primary productivity, because GPP is the product of photosynthetic LUE and APAR incident on vegetation (APAR). APAR 
is relatively well estimated using current satellite missions, because total incoming radiation to the Earth is a well-measured quantity in space and time, and atmospheric effects are known enough to calculate the radiation incident on vegetation. This section quantifies the impact of BRDF estimation errors, or their aforementioned improvement, on LUE errors and their improvement.

LUE can be correlated with the normalized difference of reflectance between a xanthophyll-insensitive reference band and the $531 \mathrm{~nm}$ band, defined as the photochemical reflectance index (PRI) [59]. Since reflectances have large angular dependence, it is easy to see how errors in reflectance measurements in either or both bands can impact their normalized difference. Inaccurate PRI leads to inaccurate LUE and therefore inaccurate GPP

$$
\mathrm{PRI}=\frac{\rho_{531 \mathrm{~nm}}-\rho_{570 \mathrm{~nm}}}{\rho_{531 \mathrm{~nm}}+\rho_{570 \mathrm{~nm}}} .
$$

Since the CAR dataset does not contain the $531 \mathrm{~nm}$ band, reflectance derived from the AMSPEC II instrument was used as truth, as detailed in Section III-B.2, for both xanthophyllsensitive $(531 \mathrm{~nm})$ and reference $(570 \mathrm{~nm})$ bands. The reflectance data was recorded over 1 hour in August 2006, timerestricted to capture the xanthophyll cycle. First-order, second moment (FOSM) propagation of uncertainty for nonlinear functions was used to statistically map the uncertainty of reflectance in either spectral band to the uncertainty of PRI. By definition of FOSM, the variance of a dependent function is a function of the variances of its variables and its partial differential with respect to them. PRI variance could therefore be represented as a function of the reflectance variance at the 531 and $570 \mathrm{~nm}$ bands

$$
\begin{aligned}
\sigma_{\mathrm{PRI}}^{2}= & \left(\left.\frac{\partial \mathrm{PRI}}{\partial \rho_{531 \mathrm{~nm}}}\right|_{\operatorname{mean}\left(\rho_{531 \mathrm{~nm}}\right)}\right)^{2} \sigma_{\rho_{531 \mathrm{~nm}}}^{2} \\
& +\left(\left.\frac{\partial \mathrm{PRI}}{\partial \rho_{682 \mathrm{~nm}}}\right|_{\operatorname{mean}\left(\rho_{682 \mathrm{~nm}}\right)}\right)^{2} \sigma_{\rho_{682 \mathrm{~nm}}}^{2} .
\end{aligned}
$$

Uncertainties in the reflectance values of the two bands were assumed to be between $21 \%$ and $31 \%$ for every angular direction, as informed by the $21 \%$ to $31 \%$ average RMS errors over all possible formation architectures using three to eight satellites [12], [48], summarized at the beginning of Section IV. Angular directional error highly correlated across wavelengths, is as seen in sensitivity studies. Since large reflectance errors for a particular angular sampling at a particular wavelength correlated with large errors for any other wavelength, given the same angular sampling, BRDF RMS error percentages were assumed to be well representative of error per angular direction (VZA-RAA combination), irrespective of wavelength. Fig. 10 shows how errors in the 522 or $570 \mathrm{~nm}$ bands (bound between $21 \%$ and $31 \%$ ) map to PRI errors using FOSM, expressed as an RMS value.

Reference PRI was assumed at -0.05 (with sign), from Section III.B.2, and its directional dependence shown in Fig. 7-right. While PRI errors increased with either band's errors, a $21 \%$ error in both bands caused a $38 \%$ error in PRI ( $\sim 0.018$ from the color map of Fig. 10) and a $31 \%$ error in the bands caused a $58 \%$ error in PRI. Unlike albedo, the error per-

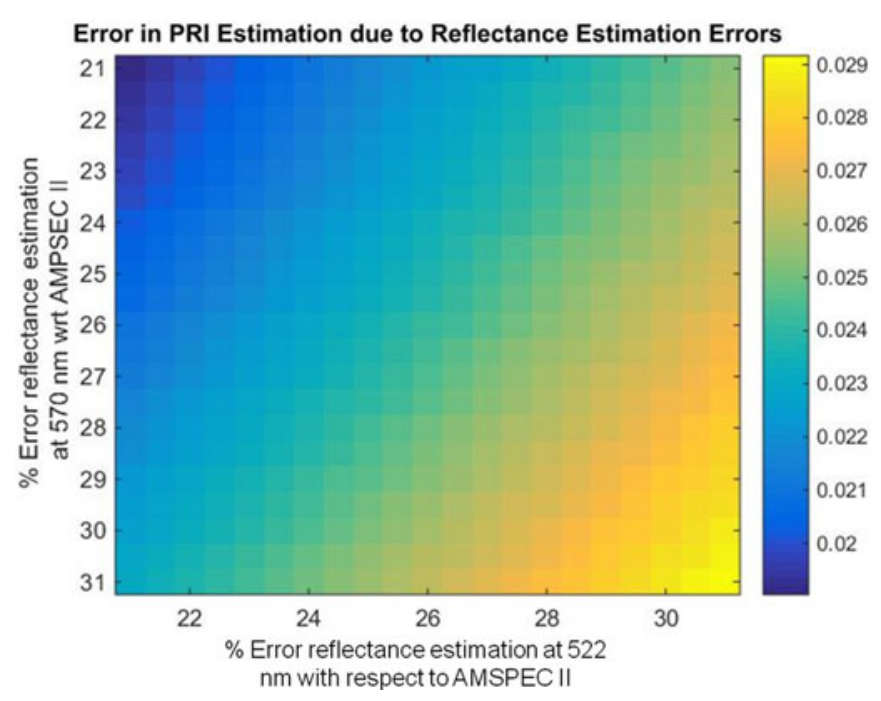

Fig. 10. PRI error (RMS value) as a function of reflection error (as \%) in the xanthophyll sensitive versus insensitive bands, calculated using firstorder second moment analysis of uncertainty. "Reference" PRI can be found in Fig. 6-right, as comparison, with a calculated mean of -0.05 .

centage of RMS PRI as a dependent BRDF product was more than the error percentage of RMS BRDF itself. This can be attributed to the additive nature of PRI with respect to reflectance or BRDF (1), where every angular value is added to another, in contrast to albedo which integrates all the angular values. Errors can also be attributed to variations in the xanthophyll cycle which would alter the reflectance at $531 \mathrm{~nm}$ over time, as well as alter the shape of the BRDF, sometimes even over a few minutes.

PRI may be mapped to LUE using data from [52], only if its angular signature has been accounted for. The authors in [52] have shown that field data when plotted between axes of PRI, without accounting for BRDF effects, versus LUE are randomly scattered. However, the same field data when plotted between axes of BRDF-corrected PRI versus LUE showed visible correlation. As seen in Fig. 11, an orange logarithmic trend-line can also be calculated. The blue cross represents BRDF-corrected reference $\operatorname{PRI}(=-0.05)$ and its corresponding $\operatorname{LUE}(=0.8)$. The gray panels are the region on the PRI axis corresponding to $-38 \%$ to $-58 \%$ error (on the left of the cross) and $+38 \%$ to $58 \%$ from reference PRI, which in turn came from the $21-31 \%$ simulated error in the reflectance bands. Due to the nonlinearity of the PRI-LUE curve, errors in PRI mapped to even more errors in LUE. Also, since the PRI-LUE plot is monotonically decreasing positive PRI errors produced negative LUE errors and vice versa. Errors around lower values of PRI (left) produced large LUE errors, compared to errors around the higher values of PRI (right). A $-38 \%$ to $-58 \%$ simulated error in PRI (about 0.05 ) mapped to $+78.7 \%$ to $143.4 \%$ simulated error in LUE. On the other hand, a $+38 \%$ to $+58 \%$ error in PRI resulted in $-54.2 \%$ to $-76.7 \%$ LUE errors.

The PRI RMS error, when estimated using multisensor, single spacecraft measurements, was $42 \%$ compared to the reference RMS PRI of 0.05 due to a multi-angular reflectance error of 23.2\% (BRDF RMS corresponding to MISR's design). If this error is positive/negative, the corresponding LUE is $0.328 / 1.518$ 


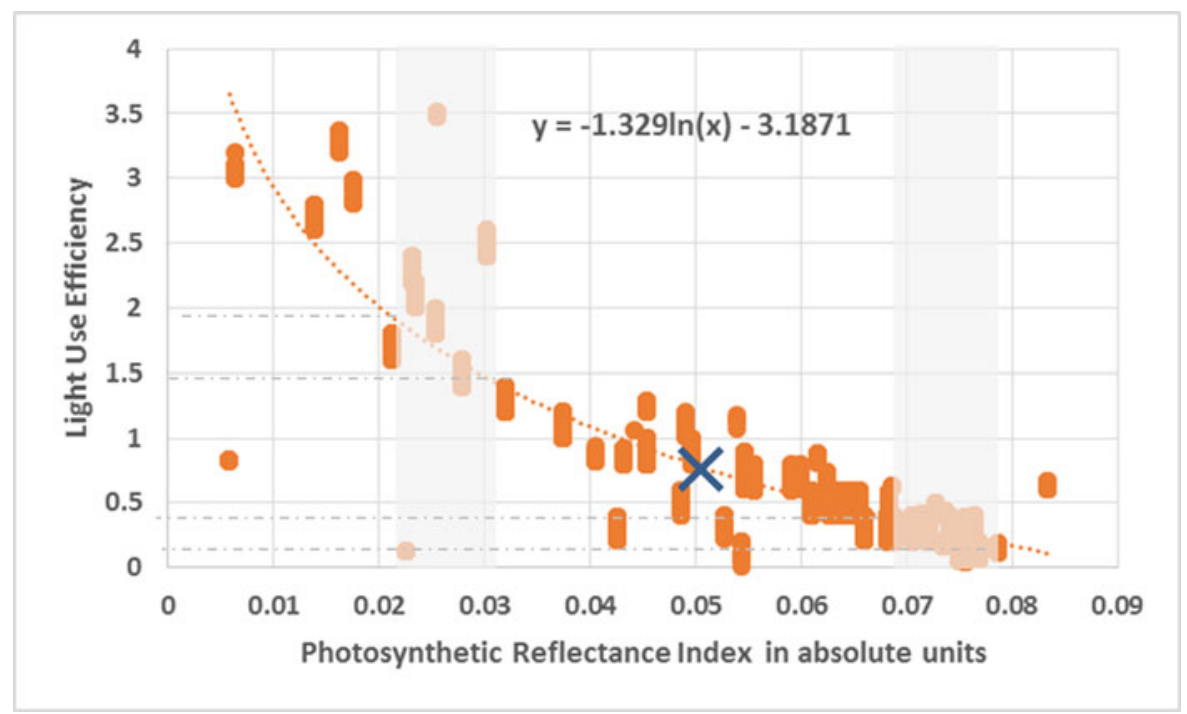

Fig. 11. Mapping between BRDF-corrected PRI and LUE (also called photosynthetic efficiency) obtained from the dataset in [52], with a fitted logarithmic trend-line. "Reference PRI" from Fig. 7-right is marked by a blue cross. Transparent grey panels mark the range of positive/negative PRI errors from Fig. 10; gray dashes mark the corresponding LUE errors.

which is $-58.9 \% /+89.8 \%$ different from the reference LUE derived from reference data in Fig. 11. In comparison, as discussed in the previous paragraph, the best case formation architecture with eight satellites produced a $38 \%$ error in RMS PRI, which mapped to a $-54.2 \% / 78.7 \%$ error in LUE. Furthermore, since LUE linearly maps to GPP for a statistically determinate APAR, the percentage of LUE error improvement between a single spacecraft (MISR arrangement) and the best formation will be reflected in GPP improvement as well.

The full tradespace of architectures, such as that for the BRDF and albedo case study, was not evaluated again, because the above analysis was deemed sufficient to establish the impact of BRDF errors on GPP estimation. The NDVI analysis presented in Section IV.C shows that it is possible that the PRI errors may be overestimated by FOSM. For example, if a 10\% error in PRI were used, in keeping with NDVI error for the best eight-satellite formation instead of the $38 \%$ predicted by FOSM, the LUE errors would be $-17.6 \% / 15.9 \%$ depending on whether the PRI error is positive/negative. Using the same method, if a 26\% PRI error were used for the multisensor, single spacecraft (MISR) arrangement, the LUE errors would be $-50.3 \% / 38.7 \%$. In fact, the improvement in LUE estimation by using a formation of eight satellites compared to single satellites was found to be even more significant if NDVI error values were to inform PRI errors instead of the FOSM theory. Moreover, the error PRI values for the formation and MISR arrangement, respectively, were very close to those obtained when Fig 7 (right) was used as reference PRI. Given the modular nature of architecture evaluation using OSSEs, if reference PRI were to be available from field campaigns as densely as CAR data, the mapping algorithm of $\{B R D F$ to PRI to LUE to GPP $\}$ can be plugged in the same way as $\{$ BRDF to albedo $\}$ and plots like Figs. 8 and 9 can be made.

\section{Impact on NDVI Estimation}

NDVI, like PRI, is a normalized difference product of reflectances and is expected to be impacted by BRDF estimation errors in a similar fashion as PRI is. Equation 3 quantifies NDVI and can be calculated using CAR band \#4 (red centred at 682 $\mathrm{nm}$ ) and \#5 (NIR centred at $870 \mathrm{~nm}$ ). NDVI Application error for every DSM architecture was computed as follows: Reference (or forward modeled) NDVI was calculated from reference (or forward modeled) BRDF at both 682 and $870 \mathrm{~nm}$, using (3) at every one of the 360 relative azimuth and 90 view zenith angles. As described in Section III-B, forward modeled BRDF was calculated from parameters estimated from the simulated angular measurements provided by any architecture. NDVI error is the difference between the reference and forward modeled $\operatorname{NDVI}(90 \times 360=32400$ values $)$. Just as BRDF error was represented as an RMS value, NDVI error is also represented as an RMS value calculated over all 32400 values

$$
\mathrm{NDVI}=\frac{\rho_{870 \mathrm{~nm}}-\rho_{682 \mathrm{~nm}}}{\rho_{870 \mathrm{~nm}}+\rho_{682 \mathrm{~nm}}} .
$$

The NDVI error, with respect to CAR reference NDVI, averaged over one day's simulation varied between 0.022 and $\sim 0.1$ for a three to eight satellite formation as shown in Fig. 12. The corresponding percentage errors ranged between $1 \%$ and $36 \%$, and were much lower than the 38-56\% error predicted for PRI using the FOSM analysis. Since NDVI and PRI have exactly the same form of equation, FOSM would have overpredicted NDVI errors. NDVI error estimated for the MISR-type arrangement of instruments on a single satellite was 0.038 or $\sim 26 \%$ difference from CAR reference NDVI. As seen in the generic BRDF case, when MATLAB's two-sample $t$-test was applied to the NDVI error time series for MISR and the best eight-satellite formation, it rejected the null hypothesis that the data are independent random samples from normal distributions with equal means and equal but unknown variances at the default $p<0.05$ significance level. Like previous BRDF products, the range of errors and the maximum error over all formation orientations decreased with increasing number of satellites. However, unlike previous products the minimum formation error did not decrease with satellite number and hardly showed 


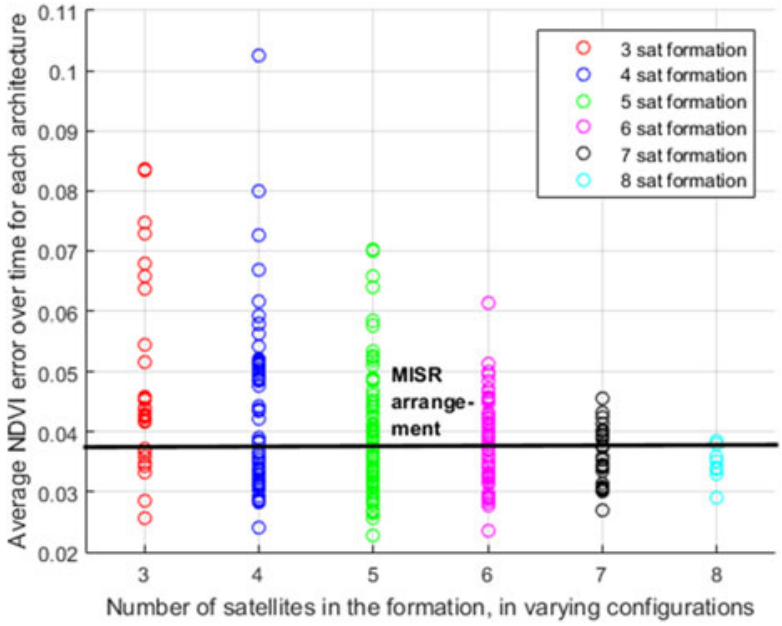

Fig. 12. Average NDVI error over time at a $650 \mathrm{~km}, 51.6^{\circ}$ orbit as the number of satellites in the DSM is increased. Each circle represents a different arrangement of the satellites in space, at the same altitude and inclination, but different planes with respect to Equatorial positioning and arrangement within the plane. The horizontal black line represents MISR's NDVI error of 0.038 as calculated in the same way as for the formations, accounting for only angular sampling.

a trend. This behavior could be due to the difference term in the NDVI equation which causes otherwise large error terms to cancel out instead of adding up. Simulations showed that a formation of eight satellites (cyan in Fig. 12) avoids the risk of inefficiently maintained formations, because all its orientations outperformed the multisensor, single spacecraft error of $0.038 \%$ or $26 \%$. The best performing eight-satellite formation averaged an NDVI error of $\sim 0.03 \%$ or $\sim 10 \%$ over time.

As seen in the albedo case, when the maximum NDVI error over all simulation time steps was considered as a metric, MISR's accuracies (compared to formations) fell further due to inflexibility in its geometric orientation causing inadequate sampling of the solar principal plane for extended periods every $\sim 45 \mathrm{~min}$. The simulated error for MISR's orbits and sensors was 0.12 while that of the minimum-error formations ranged between 0.09 and 0.11 , depending on the number of satellites in the formation. Unlike the albedo case, there were many formations whose maximum simulated NDVI error was worse than MISR's and errors went as high as 1.01 and 1.13 for the worst-case 4and 3- satellite cases, respectively. Therefore, simulations confirmed that care must be taken to select the optimal formation and invest in its optimal maintenance and operations.

\section{SUmmary DiscusSion AND Future Work}

We have demonstrated the feasibility of a formation flight solution with small satellites for space-based BRDF estimation, as well as a methodology to quantify the angular performance advantages of the proposed solution with reference to existing space and airborne missions. The advantage of using DSMs or formations is not only in improved performance with increasing satellites which can complement current, flagship missions (such as MISR) in angular coverage but also in making space systems more scalable, given uncertain budget environments. Satellites can be launched as they become available and the angular sampling and BRDF estimation will improve as more assets are in orbit. Similarly, loss of a satellite from the formation will gracefully degrade angular performance, instead of causing data discontinuity, and can be replaced at a later time.

The coupled MBSE and OSSE framework allowed the assessment of angular sampling performance in terms of BRDF and BRDF product errors, compared formation architectures against each other as well as existing multisensor, single spacecraft and verified technical feasibility of each proposed architecture in terms of existing small satellite technology to support the critical subsystems (otherwise the architectures are discarded). The systems engineering model was divided into architecture generation, architecture evaluation, and architecture sizing components. The science evaluation model used static BRDF data from existing missions binned in terms of Earth surface types as reference. It calculated the errors of forward-modeled BRDF (or dependent products) with respect to the selected reference, depending on which surface type is expected in orbit below the formation, and identified the best performing architectures. One satellite in the formation was always simulated to point nadir while the others pointed their payloads at its nadir. The nadir pointing satellite changed over time, based on a simple performance-based algorithm calculated on the ground and communicated during daily overpasses.

The proposed formations were shown to improve angular sampling (using BRDF products as metrics) of a mediumresolution ground pixel, at any given waveband of interest to the remote sensing community that needs angular signatures. Case-study specific recommendations for designing formations to estimate global BRDF and its dependent products such as albedo, LUE, NDVI, were provided. A formation of six satellites produced lesser BRDF RMS error (21.82\%), averaged over one day of simulations, compared to multisensor, single spacecraft in MISR's arrangement (23.2\%). An eight-satellite formation reduced the error to $21 \%$ at some additional cost. Albedo estimation errors were lesser than BRDF RMS errors because albedo is only a single number per multiangular image, while BRDF has an error term per angular direction in the image. The multisensor, single spacecraft albedo error of $3.6 \%$ was outperformed by a formation of three satellites $(1.86 \%)$, when arranged optimally and by a formation of five satellites (3.36\%) when arranged in any way. An eight-satellite formation was shown to be capable of pushing albedo errors to $0.67 \%$. Since the Earth's outgoing radiation is approximated as $341.3 \mathrm{~W} / \mathrm{m}^{2}$ and global albedo as 0.3 [29], less than $1 \%$ error in albedo estimation constrains the outgoing energy budget within $0.7 \mathrm{~W} / \mathrm{m}^{2}$. Currently, total outgoing radiation is accurate to only $1 \%\left(3.41 \mathrm{~W} / \mathrm{m}^{2}\right)$ and better albedo estimates can help measure uncertainties in the energy balance much better.

Photosynthetic efficiency (LUE) errors were higher than BRDF RMS errors because of the nonlinear dependence of LUE on PRI and the susceptibility of PRI to reflectance errors at 2 wavelengths, at every angular direction. The $23.3 \%$ BRDF error for MISR's arrangement in both wavebands translated to $-58.98 \%$ or $+89.77 \%$ in LUE at a $42 \%$ PRI error (depending on its sign). An optimally arranged formation architecture with eight satellites, in comparison, mapped to a $-54.22 \%$ or $78.69 \%$ of LUE error due to a 38\% PRI error. Since GPP, or the Earth's carbon budget, is proportionate to LUE as scaled by the 
relatively very well-characterized APAR, LUE improvements map to GPP improvements as percentages. Over $50 \%$ improvement in GPP errors due to formation angular sampling can improve the $40 \%$ uncertainty [36] in terrestrial carbon intake significantly. The multisensor, single spacecraft NDVI error, averaged over time, was 0.038 or $26 \%$ while the best performing eight-satellite formation provided an NDVI error of $\sim 0.03 \%$ or $\sim 10 \%$. The improvement in LUE estimation by using a formation of eight satellites $(-17.6 \% / 15.9)$ compared to multi-sensor, single spacecraft $(-50.3 \% / 38.7 \%)$ was found to be even more significant, if NDVI error values were to inform PRI errors instead of the FOSM theory (used due to the lack of BRDF reference data at PRI wavelengths). The NDVI informed errors were in keeping with those obtained from using simulated PRI as reference.

Our research has started the process of mission design for a space-based formation flight mission for multiangle remote sensing by putting high-level architectural decisions into place, and modeling the low-level components that could impact those architectural decisions. In the language of NASA's project lifecycle, it serves as a pre-Phase A level of analysis. Future work includes but is not limited to high-level payload design (to comprehensively simulate all the 3-D spectral components identified, close the design in terms of integrated optics, select the optimal imager architecture and compute expected SNR over the period of operations given internal tradeoffs such as aberrations, optics speed, number of lenses, etc. and external disturbances such as temperature, jitter, atmospheric effects, etc.), detailed analysis to identify the appropriate ADCS systems and control algorithms that meet the jitter and stability requirements imposed by the need to co-point at every instant in time and robust planning and scheduling tools (for more automation in terms of target observation, imaging mode determination and decisionmaking and downlink scheduling as a function of satellite capabilities and ground station availability).

\section{ACKNOWLEDGMENT}

The authors would like to thank Dr. W. Wiscombe (NASA GSFC) and his Leonardo-BRDF team from the 1990s who first conceptualized the use of formation flight for BRDF measurements, Dr. J. Le Moigne for important ideas in using distributed space missions for Earth observation, R. Poudyal (SSAI) for helping to extract the BRDF data used in the analysis leading to the results, and Prof. O. de Weck, Prof. K. Cahoy, and Prof. D. Miller at MIT for very useful discussions that have improved the quality of this paper.

\section{REFERENCES}

[1] F. E. Nicodemus, Geometrical Considerations and Nomenclature for Reflectance, vol. 160. Washington, DC, USA: U.S. Department of Commerce, Nat. Bureau Standards, 1977.

[2] C. K. Gatebe and M. D. King, "Airborne spectral BRDF of various surface types (ocean, vegetation, snow, desert, wetlands, cloud decks, smoke layers) for remote sensing applications," Remote Sens. Environ., vol 179, pp. 131-148, 2016

[3] C. K. Gatebe, M. D. King, S. Platnick, G. T. Arnold, E. F. Vermote, and B. Schmid, "Airborne spectral measurements of surface-atmosphere anisotropy for several surfaces and ecosystems over southern Africa," J. Geophys. Res., vol. 108, no. D13, pp. 25-1-25-16, 2003.
[4] G. T. Georgiev, C. K. Gatebe, J. J. Butler, and M. D. King, "BRDF analysis of savanna vegetation and salt-pan samples," IEEE Trans. Geosci. Remote Sens., vol. 47, no. 8, pp. 2546-2556, 2009.

[5] Z. Wang, C. A. Coburn, X. Ren, and P. M. Teillet, "Effect of soil surface roughness and scene components on soil surface bidirectional reflectance factor," Can. J. Soil Sci., vol. 92, no. 2, pp. 297-313, 2012.

[6] D. J. Diner et al., "Multi-angle imaging spectroradiometer (MISR) instrument description and experiment overview," IEEE Trans. Geosci. Remote Sens., vol. 36, no. 4, pp. 1072-1087, Jul. 1998.

[7] X. Xiong et al., "Terra and aqua modis design, radiometry, and geometry in support of land remote sensing," in Land Remote Sensing and Global Environmental Change. New York, NY, USA: Springer 2011, pp. 133164.

[8] M. J. Barnsley, J. J. Settle, M. A. Cutter, D. R. Lobb, and F. Teston, "The PROBA/CHRIS mission: A low-cost smallsat for hyperspectral multiangle observations of the earth surface and atmosphere," IEEE Trans. Geosci. Remote Sens., vol. 42, no. 7, pp. 1512-1520, Jul. 2004.

[9] A. R. Huete, C. O. Justice, and W. J. D. Van Leeuwen, "MODIS vegetation index (MOD 13). Version 3. Algorithm theoretical basis document," Greenbelt MD NASA Goddard Space Flight Cent,Greenbelt, MD, USA, vol. 7, May, 1999.

[10] S. P. Hughes and L. M. Mailhe, "A preliminary formation flying orbit dynamics analysis for Leonardo-BRDF," in Proc. IEEE Aerospace Conf., 2001, pp. 579-596.

[11] J. Esper, S. Neeck, W. Wiscombe, M. Ryschkewitsch, and J. Andary, "Leonardo-BRDF: A new generation satellite constellation," presented at the Int. Astronautical Conf., Rio de Janeiro, Brazil, 2000.

[12] S. Nag, C. K. Gatebe, and O. de Weck, "Observing system simulations for small satellite formations estimating bidirectional reflectance," Int. J. Appl. Earth Obs. Geoinform., vol. 43, pp. 102-118, 2015.

[13] C. S. Ruf et al., "The CYGNSS nanosatellite constellation hurricane mission," in Proc. IEEE Int. Geosci. Remote Sens. Symp., 2012, pp. 214-216.

[14] E. Gill, P. Sundaramoorthy, J. Bouwmeester, B. Zandbergen, and R. Reinhard, "Formation flying within a constellation of nano-satellites: The QB50 mission," Acta Astronautica, vol. 82, 2012, pp. 110-117.

[15] C. Boshuizen, J. Mason, P. Klupar, and S. Spanhake, "Results from the planet labs flock constellation," presented at the AIAA/USU Small Satellite Conf., Logan, UT, USA, 2014.

[16] K. Murthy, M. Shearn, B. D. Smiley, A. H. Chau, J. Levine, and D. Robinson, "SkySat-1: Very high-resolution imagery from a small satellite," Proc. SPIE, vol. 9241, 2014, Art. no. 92411E.

[17] J. Andrews, J. Springmann, P. Brzytwa, and C. Blake, "Spaceflight Networks-A New Paradigm for cost effective satellite communications," presented at the Small Satellite Conf., Logan, UT, USA, 2014.

[18] National Research Council, The Role of Small Satellites in NASA and NOAA Earth Observation Programs. Washington, DC, USA: National Academy Press, 2000.

[19] T. J. Sabaka, D. D. Rowlands, S. B. Luthcke, and J.-P. Boy, "Improving global mass flux solutions from Gravity Recovery and Climate Experiment (GRACE) through forward modeling and continuous time correlation," J. Geophys. Res. Solid Earth, vol. 115, 2010, Art. no. B11403.

[20] M. L. Kaiser, T. A. Kucera, J. M. Davila, O. S. Cyr, M. Guhathakurta, and E. Christian, "The STEREO mission: An introduction," in The STEREO Mission. New York, NY, USA: Springer, 2008, pp. 5-16.

[21] National Research Council, Earth Science and Applications From Space: A Midterm Assessment of NASA's Implementation of the Decadal Survey. Washington, DC, USA: National Academy Press, 2012.

[22] G. T. Arnold, S.-C. Tsay, M. D. King, J. Y. Li, and P. F. Soulen, "Airborne spectral measurements of surface-atmosphere anisotropy for Arctic sea ice and tundra," Int. J. Remote Sens., vol. 23, no. 18, pp. 3763-3781, 2002.

[23] D. Kaslow, G. Soremekun, H. Kim, and S. Spangelo, "Integrated modelbased systems engineering (MBSE) applied to the simulation of a CubeSat mission," in Proc. IEEE Aerosp. Conf., 2014, pp. 1-14.

[24] G. B. Shaw, D. Miller, and D. E. Hastings, "Development of the quantitative generalized information network analysis (GINA) methodology for satellite systems," in Proc. IEEE Aerosp. Conf., 1999, pp. 301-321.

[25] C. D. Jilla, "A multiobjective, multidisciplinary design optimization methodology for the conceptual design of distributed satellite systems," Ph.D. dissertation, Massachusetts Inst. Technol., Cambridge, MA, USA, 2002.

[26] C. P. Arnold, Jr., and C. H. Dey, "Observing-systems simulation experiments: Past, present, and future," Bull. Am. Meteorol. Soc., vol. 67, no. 6, pp. 687-695, 1986.

[27] C. K. Gatebe, C. M. Ichoku, R. Poudyal, M. O. Román, and E. Wilcox, "Surface albedo darkening from wildfires in northern sub-Saharan Africa," Environ. Res. Lett., vol. 9, no. 6, 2014, Art. no. 065003. 
[28] T. H. Vonder Haar and V. E. Suomi, "Measurements of the earth's radiation budget from satellites during a five-year period-Part I: Extended time and space means," J. Atmospheric Sci., vol. 28, no. 3, pp. 305-314, 1971.

[29] K. E. Trenberth, J. T. Fasullo, and J. Kiehl, "Earth's global energy budget," Bull. Amer. Meteorol. Soc., vol. 90, no. 3, pp. 311-323, 2009.

[30] K. E. Trenberth et al., "Challenges of a sustained climate observing system," in Climate Science for Serving Society. New York, NY, USA: Springer, 2011.

[31] A. Henderson-Sellers and M. F. Wilson, "Surface albedo data for climatic modeling," Rev. Geophys., vol. 21, no. 8, pp. 1743-1778, 1983.

[32] P. J. Sellers, "Remote sensing of the land surface for studies of global change," NASA Goddard Space Flight Center, Columbia, MD, USA, NASA/GSFC International Satellite Land Surface Climatology Project Report, 1993.

[33] J. G. Canadell et al., "Contributions to accelerating atmospheric $\mathrm{CO}_{2}$ growth from economic activity, carbon intensity, and efficiency of natural sinks," Proc. Natl. Acad. Sci., vol. 104, no. 47, pp. 18866-18870, Nov. 2007.

[34] G. R. Van der Werf et al., "CO ${ }_{2}$ emissions from forest loss," Nat. Geosci., vol. 2, no. 11, pp. 737-738, 2009

[35] M. O. Román, C. K. Gatebe, C. B. Schaaf, R. Poudyal, Z. Wang, and M. D. King, "Variability in surface BRDF at different spatial scales $(30 \mathrm{~m}-$ $500 \mathrm{~m}$ ) over a mixed agricultural landscape as retrieved from airborne and satellite spectral measurements," Remote Sens. Environ., vol. 115, no. 9, pp. 2184-2203, Sep. 2011.

[36] T. Hilker et al., "Inferring terrestrial photosynthetic light use efficiency of temperate ecosystems from space," J. Geophys. Res. Biogeosci., vol. 116, no. G3, 2011, Art. no. 32609.

[37] F. G. Hall et al., "Multi-angle remote sensing of forest light use efficiency by observing PRI variation with canopy shadow fraction," Remote Sens. Environ., vol. 112, no. 7, pp. 3201-3211, 2008.

[38] F. G. Hall, T. Hilker, and N. C. Coops, "Data assimilation of photosynthetic light-use efficiency using multi-angular satellite data: I. Model formulation," Remote Sens. Environ., vol. 121, pp. 301-308, 2012.

[39] D. C. Morton et al., "Amazon forests maintain consistent canopy structure and greenness during the dry season," Nature, vol. 506, pp. 221-224, 2014.

[40] R. B. Myneni and F. G. Hall, "The interpretation of spectral vegetation indexes," IEEE Trans. Geosci. Remote Sens. , vol. 33, no. 2, pp. 481-486, Mar. 1995.

[41] J. L. Roujean, M. Leroy, A. Podaire, and P. Y. Deschamps, "Evidence of surface reflectance bidirectional effects from a NOAA/AVHRR multitemporal data set," Int. J. Remote Sens., vol. 13, no. 4, pp. 685-698, 1992.

[42] J. Cihlar, D. Manak, and N. Voisin, "AVHRR bidirectional reflectance effects and compositing," Remote Sens. Environ., vol. 48, no. 1, pp. 7788, 1994.

[43] O. Dubovik et al., "Statistically optimized inversion algorithm for enhanced retrieval of aerosol properties from spectral multi-angle polarimetric satellite observations," Atmos. Meas. Tech., vol. 4, pp. 975-1018, 2011.

[44] O. P. Hasekamp, P. Litvinov, and A. Butz, "Aerosol properties over the ocean from PARASOL multiangle photopolarimetric measurements," J. Geophys. Res. Atmos., vol. 116, no. D14, 2011, Art. no. D14204.

[45] M. I. Mishchenko et al., "Accurate monitoring of terrestrial aerosols and total solar irradiance: Introducing the Glory Mission," Bull. Amer. Meteorol. Soc., vol. 88, no. 5, pp. 677-691, 2007.

[46] C. Fish, A. Marchant, E. Stromberg, and S. Sullivan, "High performance spectroscopic observation from a CubeSat," presented at the AIAA/USU Conf. Small Satellite, Logan, UT, USA, 2013.

[47] R. Shishko and R. Aster, "NASA systems engineering handbook," NASA Special Publication, vol. 6105, 1995.

[48] Sreeja Nag, "Design and evaluation of distributed spacecraft missions for multi-angular earth observation," Ph.D. dissertation, Massachusetts Inst. Technol., Cambridge, MA, USA, 2015.

[49] S. Nag, C. Gatebe, D. W. Miller, and O. L. De Weck, "Effect of satellite formation architectures and imaging modes on global albedo estimation," Acta Astronautica, vol. 126, pp. 77-97, 2016.

[50] J.-L. Roujean, M. Leroy, and P.-Y. Deschamps, "A bidirectional reflectance model of the Earth's surface for the correction of remote sensing data," J. Geophys. Res. Atmos., vol. 97, no. D18, pp. 20455-20468, 1992.

[51] C. Cox and W. Munk, "Statistics of the sea surface derived from sun glitter," J Marine Res., vol. 13, no. 2, pp. 198-227, 1954.

[52] T. Hilker et al., "Separating physiologically and directionally induced changes in PRI using BRDF models," Remote Sens. Environ., vol. 112, no. 6, pp. 2777-2788, 2008.

[53] T. Hilker et al., "Remote sensing of photosynthetic light-use efficiency across two forested biomes: Spatial scaling," Remote Sens. Environ., vol. 114, no. 12, pp. 2863-2874, Dec. 2010.
[54] M. D. King, M. Strange, P. Leone, and L. Blaine, "Multiwavelength scanning radiometer for airborne measurements of scattered radiation within clouds," J. Atmospheric Ocean. Technol., vol. 3, pp. 513-522, 1986.

[55] C. Godsalve, "Bi-directional reflectance sampling by ATSR-2: A combined orbit and scan model," Remote Sens., vol. 16, no. 2, pp. 269-300, 1995.

[56] M. Abrams, "The Advanced Spaceborne Thermal Emission and Reflection Radiometer (ASTER): Data products for the high spatial resolution imager on NASA's Terra platform," Int. J. Remote Sens., vol. 21, no. 5, pp. 847859, 2000.

[57] B. A. Wielicki, B. R. Barkstrom, E. F. Harrison, R. B. Lee, G. L. Smith, and J. E. Cooper, "Clouds and the Earth's Radiant Energy System (CERES): An earth observing system experiment," Bull. Amer. Meteorol. Soc., vol. 77, no. 5, pp. 853-868, 1996.

[58] P. Y. Deschamps et al., "The POLDER mission: Instrument characteristics and scientific objectives," IEEE Trans. Geosci. Remote Sens., vol. 32, no. 3, pp. 598-615, May 1994.

[59] T. Hilker et al., "Separating physiologically and directionally induced changes in PRI using BRDF models," Remote Sens. Environ., vol. 112, pp. 2777-2788, 2008.

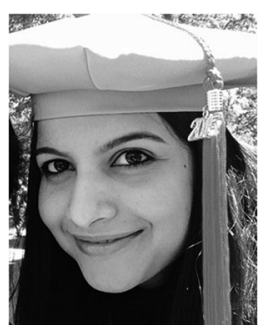

Sreeja Nag received the Ph.D. degree in space systems (Engineering), the M.S. degree in aeronautics and astronautics and the M.S. degree in technology and policy, all from the Massachusetts Institute of Technology, Cambridge, MA, USA. She has a B.S. and M.S. in Exploration Geophysics from the Indian Institute of Technology, Kharagpur, India.

She is currently a Research Scientist and Engineer with the Bay Area Environmental Research Institute, based at NASA Ames Research Center and NASA Goddard Space Flight Center. She is a Principal Investigator or Co-Investigator in several NASA funded grants in the areas of constellations and formation flight design for Earth science products. She is also a Member of the NASA Unmanned Air Vehicle (UAV) Traffic Management team, where she works on communication and navigation for distributed UAV systems. She led the SPHERES Zero Robotics program from 2011 to 2012 and has worked as an International Fellow in the European Space Agency in 2010.

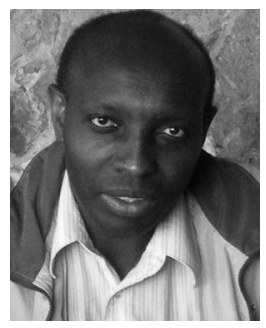

Charles K. Gatebe received the B.Sc. degrees in meteorology, mathematics, and physics, and the M.Sc. degree in meteorology, all from the University of Nairobi, Kenya, in 1990 and 1994, respectively, and the Ph.D. degree in atmospheric sciences from the University of Witwatersrand, South Africa, in 1999.

He is currently working with the Universities Space Research Association based at NASA Goddard Space Flight Center, Climate and Radiation Laboratory, Greenbelt, MD, USA. He is a Group Leader and the Principal Investigator of NASA's Cloud Absorption Radiometer instrument. He is a Leader in the airborne measurements and analysis of surface bidirectional reflectance distribution function, which is needed to study and advance the knowledge of clouds, aerosols, ecosystem structure and function, albedo, and feedbacks to climate. In addition, he has a wide range of experience in calibration of scanning radiometers, development of new remote sensing instruments and participated in numerous NASA-led airborne field experiments.

Dr. Gatebe has received many awards including the prestigious Robert $\mathrm{H}$. Goddard Award for Outreach and the World Meteorological Organization Young Scientist Award.

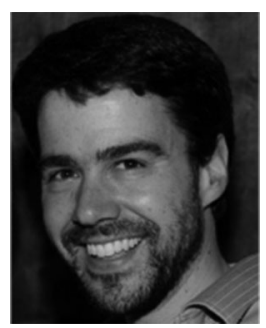

Thomas Hilker received the B.S. degree in forestry from the University of Applied Sciences Goettingen, Hildesheim, Germany, the Master's degree in photogrammetry and geoinformatics from the University of Applied Sciences Stuttgart, Stuttgart, Germany, and the Ph.D. degree in forestry from the University of British Columbia, Vancouver, BC, USA, in 2000, 2002, and 2008, respectively.

$\mathrm{He}$ is currently an Associate Professor at the Department of Geography and Environment, in Southampton, United Kingdom. His research interest includes remote sensing of the carbon, water, and energy balance of terrestrial ecosystems. He uses remotely sensed observations from towers to satellites to obtain information about vegetation carbon uptake over land and relate this data to models of the carbon, water and energy cycle regionally and globally. 